\title{
Rapid antigen tests for dengue virus serotypes and Zika virus in patient serum
}

Irene Bosch, ${ }^{1,2 *}$ Helena de Puig, ${ }^{1,3 *}$ Megan Hiley, ${ }^{1}$ Marc Carré-Camps, ${ }^{1,4}$ Federico Perdomo-Celis, ${ }^{5}$ Carlos F. Narváez, ${ }^{5}$ Doris M. Salgado, ${ }^{5}$ Dewahar Senthoor, ${ }^{1}$ Madeline O'Grady, $^{1}$ Elizabeth Phillips, ${ }^{1}$ Ann Durbin, ${ }^{1,6}$ Diana Fandos,, ${ }^{1,4}$ Hikaru Miyazaki, ${ }^{1}$ Chun-Wan Yen, ${ }^{1}$ Margarita Gélvez-Ramírez, ${ }^{7}$ Rajas V. Warke, ${ }^{8}$ Lucas S. Ribeiro, ${ }^{9}$ Mauro M. Teixeira, ${ }^{9}$ Roque P. Almeida, ${ }^{10}$ José E. Muñóz-Medina, ${ }^{11}$ Juan E. Ludert, ${ }^{12}$ Mauricio L. Nogueira, ${ }^{13}$ Tatiana E. Colombo, ${ }^{13}$ Ana C. B. Terzian, ${ }^{13}$ Patricia T. Bozza, ${ }^{14}$ Andrea S. Calheiros, ${ }^{14}$ Yasmine R. Vieira, ${ }^{15}$ Giselle Barbosa-Lima, ${ }^{15}$ Alexandre Vizzoni, ${ }^{15}$ José Cerbino-Neto, ${ }^{15}$ Fernando A. Bozza, ${ }^{15,16}$ Thiago M. L. Souza, ${ }^{14,17}$ Monique R. O. Trugilho, ${ }^{18}$ Ana M. B. de Filippis, ${ }^{19}$ Patricia C. de Sequeira, ${ }^{19}$ Ernesto T. A. Marques, $^{20,21}$ Tereza Magalhaes, $^{20,22}$ Francisco J. Díaz, ${ }^{23}$ Berta N. Restrepo, ${ }^{24}$ Katerine Marín, $^{24}$ Salim Mattar, ${ }^{25}$ Daniel Olson, ${ }^{26}$

Edwin J. Asturias, ${ }^{26}$ Mark Lucera, ${ }^{27}$ Mohit Singla, ${ }^{28}$ Guruprasad R. Medigeshi, $^{29}$

Norma de Bosch, ${ }^{30}$ Justina Tam, ${ }^{1,31}$ Jose Gómez-Márquez, ${ }^{1}$ Charles Clavet, ${ }^{31}$

Luis Villar, ${ }^{7}$ Kimberly Hamad-Schifferli, ${ }^{3,32+}$ Lee Gehrke ${ }^{1,33+}$

The recent Zika virus (ZIKV) outbreak demonstrates that cost-effective clinical diagnostics are urgently needed to detect and distinguish viral infections to improve patient care. Unlike dengue virus (DENV), ZIKV infections during pregnancy correlate with severe birth defects, including microcephaly and neurological disorders. Because ZIKV and DENV are related flaviviruses, their homologous proteins and nucleic acids can cause cross-reactions and false-positive results in molecular, antigenic, and serologic diagnostics. We report the characterization of monoclonal antibody pairs that have been translated into rapid immunochromatography tests to specifically detect the viral nonstructural 1 (NS1) protein antigen and distinguish the four DENV serotypes (DENV1-4) and ZIKV without cross-reaction. To complement visual test analysis and remove user subjectivity in reading test results, we used image processing and data analysis for data capture and test result quantification. Using a 30- $\mu$ l serum sample, the sensitivity and specificity values of the DENV1-4 tests and the pan-DENV test, which detects all four dengue serotypes, ranged from 0.76 to 1.00 . Sensitivity/specificity for the ZIKV rapid test was $0.81 / 0.86$, respectively, using a $150-\mu$ I serum input. Serum ZIKV NS1 protein concentrations were about 10-fold lower than corresponding DENV NS1 concentrations in infected patients; moreover, ZIKV NS1 protein was not detected in polymerase chain reactionpositive patient urine samples. Our rapid immunochromatography approach and reagents have immediate application in differential clinical diagnosis of acute ZIKV and DENV cases, and the platform can be applied toward developing rapid antigen diagnostics for emerging viruses.

\section{INTRODUCTION}

Genus Aedes mosquitoes transmit dengue virus (DENV) and Zika virus (ZIKV), which are related flaviviruses and important global pathogens that inflict a heavy burden on public health systems. Dengue has a wide distribution, with more than a quarter of the world's population at risk and hundreds of millions of new infections annually. There are four DENV serotypes, and infection with one of the four DENV serotypes fails to provide long-lasting immunity against the remaining three viral serotypes. Some reports suggest that disease severity varies among DENV serotypes (1). ZIKV gained global attention in 2015 when thousands of clinical cases appeared suddenly in northern Brazil, with accompanying reports of devastating congenital defects, including microcephaly and Guillain-Barré syndrome. The World Health Organization subsequently declared ZIKV a global public health emergency.

Cost-effective diagnostics are urgently needed to detect and distinguish DENV and ZIKV, as well as other pathogenic viruses. The flavivirus nonstructural 1 (NS1) protein is a useful infection marker because of its release from infected cells into the bloodstream and its accumulation in dengue patients at concentrations up to $50 \mu \mathrm{g} / \mathrm{ml}(2)$. A number of commercial DENV NS1 rapid tests have been reported and compared (3-9). However, these studies were performed before the ZIKV epidemic, and a recent publication confirms DENV-ZIKV NS1 cross-reactivity using a commercial rapid test (10). In addition, none of the commercial rapid tests distinguishes the DENV serotypes.

Combining NS1 with immunoglobulin M (IgM) and IgG detection in a dual test improves sensitivity and specificity for DENV compared to NS1 alone $(3,4,8)$. However, the primary antigen for IgG and IgM during flavivirus infections is the viral envelope (E) protein, and the similarity of the flavivirus envelope proteins fuels additional problems with cross-reactivity and false-positive test results (11). There are currently no approved or widely used vaccines to prevent ZIKV or DENV infections nor low-cost rapid antigen-based diagnostics demonstrated to identify ZIKV infections without cross-reactive interference of related DENVs. Here, we describe viral NS1 antigen-based rapid tests that use monoclonal antibody (mAb) pairs to detect and distinguish the four DENV serotypes, as well as ZIKV, without cross-reactivity.

\section{RESULTS}

Approach for developing a rapid diagnostic platform to detect viral antigens

The stepwise approaches used to develop antigen-based rapid diagnostics are shown in fig. S1 and table S1. The percentages of amino 
acid homology and identity among flavivirus NS1 proteins are high (table S2); therefore, we reasoned that extensive and strategic screening would be required to identify mAbs that detect and distinguish the viruses in a rapid diagnostic test. We tested several commercial antidengue NS1 antibodies available from different vendors but found that native NS1 protein was not recognized or that there was clearly identifiable cross-reactive binding among the DENV serotype NS1 proteins. We therefore generated and characterized anti-NS1 antibodies (fig. S1 and table S1). Groups of mice were injected separately with DENV1-4 recombinant NS1 (rNS1) protein or with ZIKV rNS1 protein. B cells from the spleen or lymph nodes were fused with mouse myeloma cells to generate hybridomas. Initial hybridoma screening (209 DENV hybridomas and 104 ZIKV hybridomas) was performed by enzymelinked immunosorbent assay (ELISA) using individual rNS1 protein as antigen (step 1; table S1). By screening the hybridoma supernatants against individual DENV1-4 NS1 or ZIKV NS1 proteins, the relative ELISA values provided an initial evaluation of differential binding properties for each antibody.

Because of the similarities among the DENV NS1 and ZIKV NS1 proteins (table S2), each group of mice immunized with a single purified rNS1 protein yielded a pool of antibodies, showing both selective binding to a single DENV serotype or ZIKV NS1 and cross-reactive DENV and ZIKV antibodies (fig. S2). ZIKV NS1 hybridomas produced from lymph node tissue represented a higher proportion of clones, showing minimal cross-reactivity with dengue NS1 or other flavivirus NS1 proteins, as compared to spleen cell hybridomas (fig. S2). Using the relative ELISA values, $11 \mathrm{DENV} \mathrm{mAbs}$ and $10 \mathrm{ZIKV} \mathrm{mAbs}$ were selected for further analysis. In step 2 (fig. S1 and table S1), hybridoma supernatants were used to stain permeabilized Vero cells that had been infected with known ZIKV or DENV viral serotypes. Flow cytometric analysis demonstrated that the mAbs recognized native NS1 protein expressed by virus-infected cells and provided a quantitative analysis of cross-reactive binding when ZIKV antibodies were used to stain DENV-infected cells and vice versa. The 11 DENV mAbs and $10 \mathrm{ZIKV}$ $\mathrm{mAbs}$ recognized NS1 protein present in the virus-infected cells; therefore, the hybridomas were expanded, and the mAb isotypes were defined in preparation for affinity chromatography purification.

The purified antibodies were tested in immunochromatography pairs (step 3; fig. S1 and table S1), with one antibody conjugated to gold nanoparticles and one antibody adsorbed to nitrocellulose membrane. The 11 DENV hybridomas were tested in a matrix for interactions with DENV NS1 serotypes 1 to 4, ZIKV NS1, or without added antigen as a control. The $10 \mathrm{ZIKV}$ mAbs were also tested in a matrix using ZIKV NS1, a mixture of the four DENV serotype NS1 proteins, or no antigen as a control. We tested $726 \mathrm{DENV}$ combinations $(11 \times$ $11 \times 6=726)$ and $300 \mathrm{ZIKV}$ combinations $(10 \times 10 \times 3=300)$. Testing throughput was increased by using the half-strip dipstick format (12), where dipsticks are run in rapid format (about $20 \mathrm{~min}$, depending on humidity conditions) by placing them in microcentrifuge tubes containing small-volume suspensions of conjugated nanoparticles and sample without need for sample paper pads and conjugate paper pads that are characteristic of lateral flow chromatography. The DENV and ZIKV matrices and the immunochromatography results are shown in table S3. Eight DENV mAbs and two ZIKV mAbs were ultimately incorporated into the rapid tests used to analyze patient samples. The $10 \mathrm{mAb}$ names, their relative NS1 recognition values in initial screening, and the final application in the rapid tests are shown in Fig. 1A and table S4.

\section{Linear peptide epitope mapping: Mechanisms of specific mAb-NS1 interactions}

To begin to define mechanisms of specific mAb-NS1 interactions, we performed linear peptide epitope mapping (step 4; fig. S1 and table S1). Libraries of tiled DENV NS1 peptides were spotted onto nitrocellulose membranes and incubated with antibody. After washing, positive signals were detected using an anti-mouse IgG antibody coupled to horseradish peroxidase for signal development. In a second approach, tiled peptides were synthesized on glass slides and incubated with each of the antibodies. Positive signals were detected by immunofluorescence microscopy and were scored. The epitope mapping data are summarized in Fig. 1. mAb 7724.323 (“323”) fits the definition of a pan-DENV NS1 antibody because it recognizes all four serotype NS1 proteins in the epitopes 109 to 124 amino acid region. Six antibodies used in the rapid tests (using suffix nomenclature only: $55,323,1,130,110$, and 271) recognize the amino acids 109 to 124 epitope of the "wing" domain (amino acids 30 to 180) $(13,14)$. However, with the exception of a few pairs with mAb 243, these six antibodies do not recognize the ZIKV NS1 protein (Fig. 1B and table S3). The epitope mapping helped to explain the observed antibody specificity

\footnotetext{
${ }^{1}$ Institute for Medical Engineering and Science, Massachusetts Institute of Technology, Cambridge, MA 02139, USA. ${ }^{2}$ Department of Medicine, Mount Sinai School of Medicine, New York, NY 10029, USA. ${ }^{3}$ Department of Mechanical Engineering, Massachusetts Institute of Technology, Cambridge, MA 02139, USA. Institut Químic de Sarrià, Universitat Ramon Llull, Barcelona, Spain. ${ }^{5}$ Programa de Medicina, Facultad de Salud, Universidad Surcolombiana, Neiva, Colombia. ${ }^{6}$ Program in Virology, Division of Medical Sciences, Harvard Medical School, Boston, MA 02115, USA. 'Universidad Industrial de Santander and AEDES Program (Aliarza para el desarrollo de estrategias que disminuyan el impacto de enfermedades transmitidas por Aedes como resultado del estudo de sus endemias y epidemias), Bucaramanga, Santander, Colombia. ${ }^{8}$ HiMedia Laboratories Pvt. Ltd., Mumbai, India. ${ }^{9}$ Immunopharmacology Group, Instituto de Ciências Biológicas, Universidde Federal de Minas Gerais, Avenida Antônio Carlos 6627, Belo Horizonte, Bazil. ${ }^{10}$ Departamento de Medicina Interna e Patologia, Hospital Universitário/Empresa Brasileira de Serviços Hospitalares (EBSERH), Universialde Federal de Sergipe, Aracaju, Brazil. ${ }^{11}$ Laboratorio Central de Epidemiología, Instituto Mexicano del Seguro Social, Avenida Jacarandas S/N, Esquina Circuito Interior, Colonia La Raza Del Azcapotzalco, Códjio Postal 02990 México D.F., México. ${ }^{12}$ Departamento de Infectómica y Patogénesis Molecular, Centro de Investigacón y de Estudios Avanzados del Instituto Politécnico Nacional (CINVESTAV-IPN), Ciudad de México, México. ${ }^{13}$ Faculdade de Medicina de São José do Rio Preto (FAMERP), São José do Rio Preto, Brazil. ${ }^{14}$ Immunopharmacology Laboratory, Oswaldo Cruz Foundation (FIOCRUZ), Rio de Janeiro, Brazil. ${ }^{15}$ National Institute of Infectious Disease Evando Chagas, FIOCRUZ, Rio de Janeiro, Brazil. ${ }^{16} \mathrm{D}^{\prime}$ Or Institute of Research and Education (IDOR), Rio de Janeiro, Brazil. ${ }^{17}$ National Institute for Science and Technology on Innovation on Neglected Dseases (INCT/IDN), Center for Technological Development in Health (CD B), FIOCRUZ, Rio de Janeiro, Brazil. ${ }^{18}$ Toxinology Laboratory and Center for Technological Devebpment in Health (CDTS), FIOCRUZ, Rio de Janeiro, Brazil. ${ }^{19}$ Flavivirus Laboratory, FIOCRUZ, Rio de Janeiro, Brazil. ${ }^{20}$ Aggeu Magalhães Research Center, FIOCRUZ, Pernambuco, Recife, Brazil..2 Department of Infectious Disease and Microbiology, University of Pittsburgh, Pittsburgh,

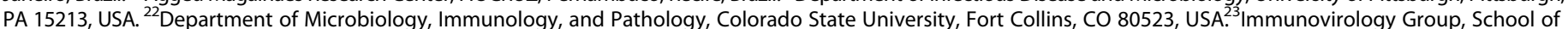
Medicine, University of Antioquia, Medellín, Colombia. ${ }^{24}$ Instituto Colombiano de Medicina Tropical (ICMT), Universidad CES, Sabaneta, Antioquia, Colombia. ${ }^{25}$ Universidad de Córdoba, Montería, Córdoba, Colombia. ${ }^{26}$ Division of Infectious Diseases, Department of Pediatrics, University of Colorado School of Medicine, Aurora, CO 80045, USA. ${ }^{27}$ Division of Infectious Diseases, Department of Medicine, University of Colorado School of Medicine, Aurora, CO 80045, USA. ${ }^{28}$ Department of Paediatrics, All India Institute of Medical Sciences, Ansari Nagar, New Delhi, India. ${ }^{29}$ Translational Health Science and Technology Institute, Faridabad, India. ${ }^{30}$ Universidad Central de Venezuela, Caracas, Venezuela. ${ }^{31}$ Winchester Engineering Analytical Center (WEAC), Winchester, MA 01890, USA. ${ }^{32}$ Department of Engineering, University of Massachusetts Boston, Boston, MA 02125, USA. ${ }^{33}$ Department of Microbiology and Immunobiology, Harvard Medical School, Boston, MA 02115, USA.

*These authors contributed equally to this work.

tCorresponding author. Email: kim.hamad@umb.edu (K.H.-S.); Igehrke@mit.edu (L.G.)
} 
A NS1 protein linear epitope recognition by the ten antibodies used in rapid test

\begin{tabular}{|c|c|c|c|c|}
\hline Antibody & Use in rapid test & Linear epitope & Position & Structure \\
\hline MA724.271 & $\begin{array}{c}\text { Membrane, dipstick } 1 \text { (DENV1) } \\
\text { nanoparticles, dipstick } 5 \text { (pan-DENV) }\end{array}$ & DENV3 NS1: MELKYSWKTWGLAKIVT & 109 & Wing \\
\hline MA7729.912 & Nanoparticles, dipstick 1 (DENV1) & DENV1 NS1:|PRIYGGPISQHNYR & 243 & $\beta$-Ladder \\
\hline MA7729.1 & Membrane, dipstick 2 (DENV2) & $\begin{array}{l}\text { DENV1 NS1 : MEHKYSWKSWGKAKII } \\
\text { DENV1 NS1 : IPKIYGGPISQHNYR }\end{array}$ & $\begin{array}{l}109 \\
243\end{array}$ & $\begin{array}{c}\text { Wing } \\
\beta \text {-Ladder }\end{array}$ \\
\hline MA7732.243 & $\begin{array}{c}\text { Nanoparticles, dipstick } 2 \text { (DENV2) } \\
\text { nanoparticles, dipstick } 5 \text { (pan-DENV) }\end{array}$ & DENV2 NS1: CRSCTLPPLRYRGEDGCW & 318 & $\beta$-Ladder \\
\hline MA724.55 & $\begin{array}{l}\text { Membrane, dipstick } 3 \text { (DENV3) } \\
\text { membrane, dipstick } 4 \text { (DENV4) }\end{array}$ & $\begin{array}{l}\text { DENV1 NS1: IPKIYGGPISQHNYR } \\
\text { DENV3 NS1: MELKYSWKTWGLAKIVT } \\
\text { DENV3.NS1: GVFTTNIWLKLREVYTQ } \\
\text { DENV4.NS1: GFGMFTTNIWMKFREG }\end{array}$ & $\begin{array}{l}243 \\
109 \\
161 \\
159\end{array}$ & $\begin{array}{l}\beta \text {-Ladder } \\
\text { Wing } \\
\text { Wing/finger } \\
\text { Wing/finger }\end{array}$ \\
\hline MA724.411 & $\begin{array}{c}\text { Nanoparticles, dipstick } 3 \text { (DENV3) } \\
\text { nanoparticles, dipstick } 5 \text { (pan-DENV) }\end{array}$ & $\begin{array}{l}\text { DENV1 NS1: : PRKIYGGPISQHNYR } \\
\text { DENV3 NS1: MELKYSWKTWGLAKIVT } \\
\text { DENV1 NS1: IWLKLRDSYTQMCDH }\end{array}$ & $\begin{array}{l}243 \\
109 \\
167\end{array}$ & $\begin{array}{c}\beta \text {-Ladder } \\
\text { Wing } \\
\text { Wing/finger }\end{array}$ \\
\hline MA725.626 & $\begin{array}{c}\text { Nanoparticles, dipstick } 4 \text { (DENV4) } \\
\text { nanoparticles, dipstick } 5 \text { (pan-DENV) }\end{array}$ & $\begin{array}{l}\text { DENV3 NS1: MELKYSWKTWGLAKIVT } \\
\text { DENV4 NS1: GFGMFTTNIWMKFREG } \\
\text { DENV4 NS1: MPPLRFLGEDGCWYGME }\end{array}$ & $\begin{array}{l}109 \\
159 \\
318\end{array}$ & $\begin{array}{c}\text { Wing } \\
\text { Wing/finger } \\
\beta \text {-Ladder }\end{array}$ \\
\hline MA724.323 & Membrane, dipstick 5 (pan-DENV) & $\begin{array}{l}\text { DENV1 NS1: MEHKYSWKSWGKAKII } \\
\text { DENV2 NS1: TELKYSWKTWGKAKML } \\
\text { DENV3 NS1: MELKYSWKTWGLAKIVT } \\
\text { DENV3 NS1: GVFTNIWIWLKLREVYQ } \\
\text { DENV4 NS1: PVNDLKYSWKTWGKAKI }\end{array}$ & $\begin{array}{l}109 \\
114 \\
109 \\
161 \\
107\end{array}$ & $\begin{array}{l}\text { Wing } \\
\text { Wing } \\
\text { Wing } \\
\text { Wing/finger } \\
\text { Wing }\end{array}$ \\
\hline $7746-50.110$ & Membrane, ZIKV NS1 dipstick & $\begin{array}{l}\text { ZIKV NS1: KECPLKHRAWNSFLV } \\
\text { ZIKV NS1: LSFRAKDGCWYGMEI }\end{array}$ & $\begin{array}{l}141 \\
321\end{array}$ & $\begin{array}{c}\text { Wing } \\
\beta \text {-Ladder }\end{array}$ \\
\hline $7746-50.130$ & Nanoparticles, ZIKV NS1 dipstick & ZIKV NS1: NELPHGWKAWGKSYF & 109 & Wing \\
\hline
\end{tabular}

Fig. 1. NS1 protein alignment and linear epitope mapping of the $\mathbf{1 0}$ antibodies used to run the DENV serotype-specific NS1 rapid tests, pan-DENV NS1 test, and ZIKV NS1 test. (A) Table listing mAb names, mAb immunochromatography applications, mAb linear epitope sequences and starting amino acid positions, and NS1 domain positions. (B) Comparison of amino acid similarity based on analysis of NS1 protein sequences from the following viruses: DENV1 (strain Singapore/S275/1990), accession number P33478; DENV2 (strain New Guinea C), accession number AAA42941; DENV3 (strain Philippines/H87/1956), accession number AAA99437; DENV4 (strain Singapore/8976/1995), accession number AAV31422; ZIKV, accession number KU497555.1. Amino acid sequences were compared using Color Align Conservation www.bioinformatics.org/sms2/color_align_cons.html to enhance the output of sequence alignment program. Residues that are identical among the sequences are boxed. Linear peptide epitopes (B) are italicized and indicated in color in the figure, with the key to the right of the figure.

in the rapid tests. The shared DENV1-4 NS1 region D/ELKYSWKTWG (amino acids 110 to 119) (15) is not conserved in ZIKV NS1; rather, the comparable ZIKV NS1 region has a distinct amino acid sequence (Fig. 1B), explaining how DENV NS1 mAbs, represented by the members of the 1/55/110/130/271/323 mAb group, do not cross-react with the ZIKV NS1 protein. mAb 243, which showed high specificity for DENV2 (tables S3 and S4), recognized peptides 313 to 330 in DENV2 NS1 (Fig. 1B). The screening approaches, combined with the epitope mapping, contributed to selecting antibodies representing positional epitope diversity; that is, epitopes in both the wing/wing connector/ finger and $\beta$-ladder domains (Fig. 1).

Matrix-based screening (table S3) revealed that the following pairs (nanoparticle/membrane) had optimal rapid test specificity: DENV1, 912/271; DENV2, 243/1; DENV3, 411/55; DENV4, 626/55. In addition, the 912 and 243 antibodies displayed excellent single serotype specificity for the DENV1 and DENV2 proteins, respectively. These antibodies might be predicted to work as 912/912 and 243/243 homopairs in detecting DENV1 and DENV2 NS1 dimers. However, experimental results showed that the limits of NS1 detection were improved by using hetero-pairs without sacrificing specificity; therefore, 912 was paired with 271, and 243 was paired with 1. For ZIKV NS1 detection, we used the 130/110 mAb pair, where both antibodies arose from the lymph node tissue approach (fig. S2). The 411, 55, and 626 antibodies did not display the single serotype specificity observed with 912 and 243
B NS1 protein alignment and epitope mapping of the 10 antibodies used in rapid tests

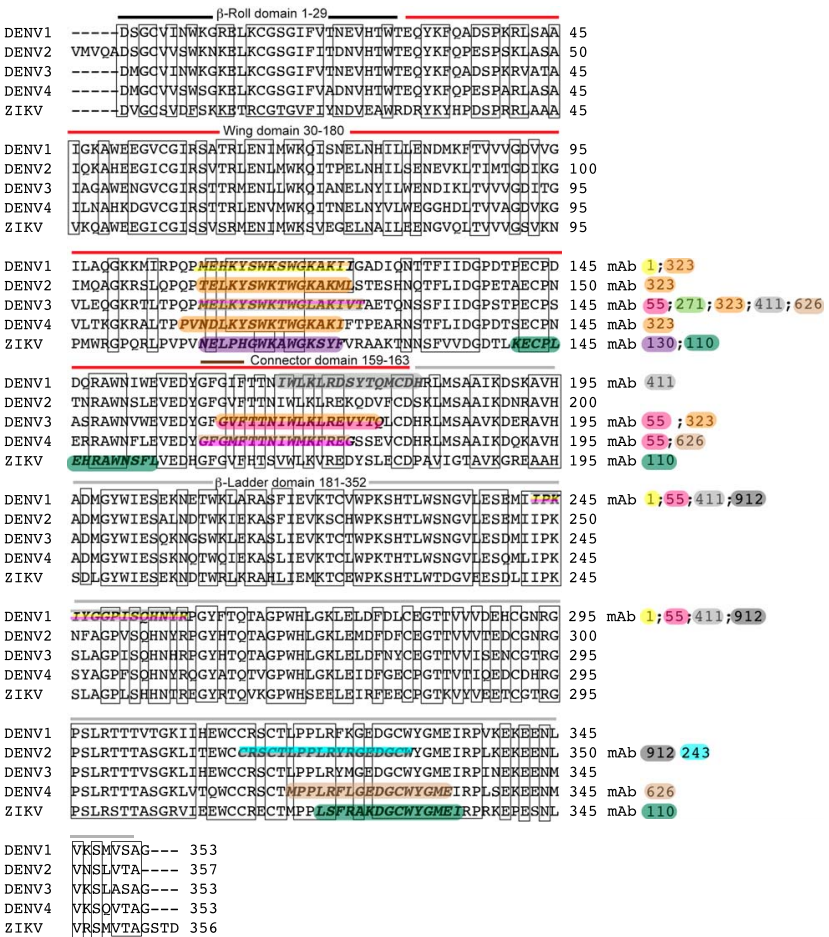

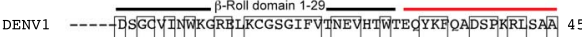

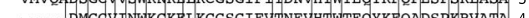

DENV4 ----DMGCYVSWSGKBLKCGSGIFVADNVHTW TEOYKFOPESPARLASA 45

$1 ; 323 ; 411 ; 626$

$195 \mathrm{mAb} 55 ; 323$

VKSLASAG-- 
A
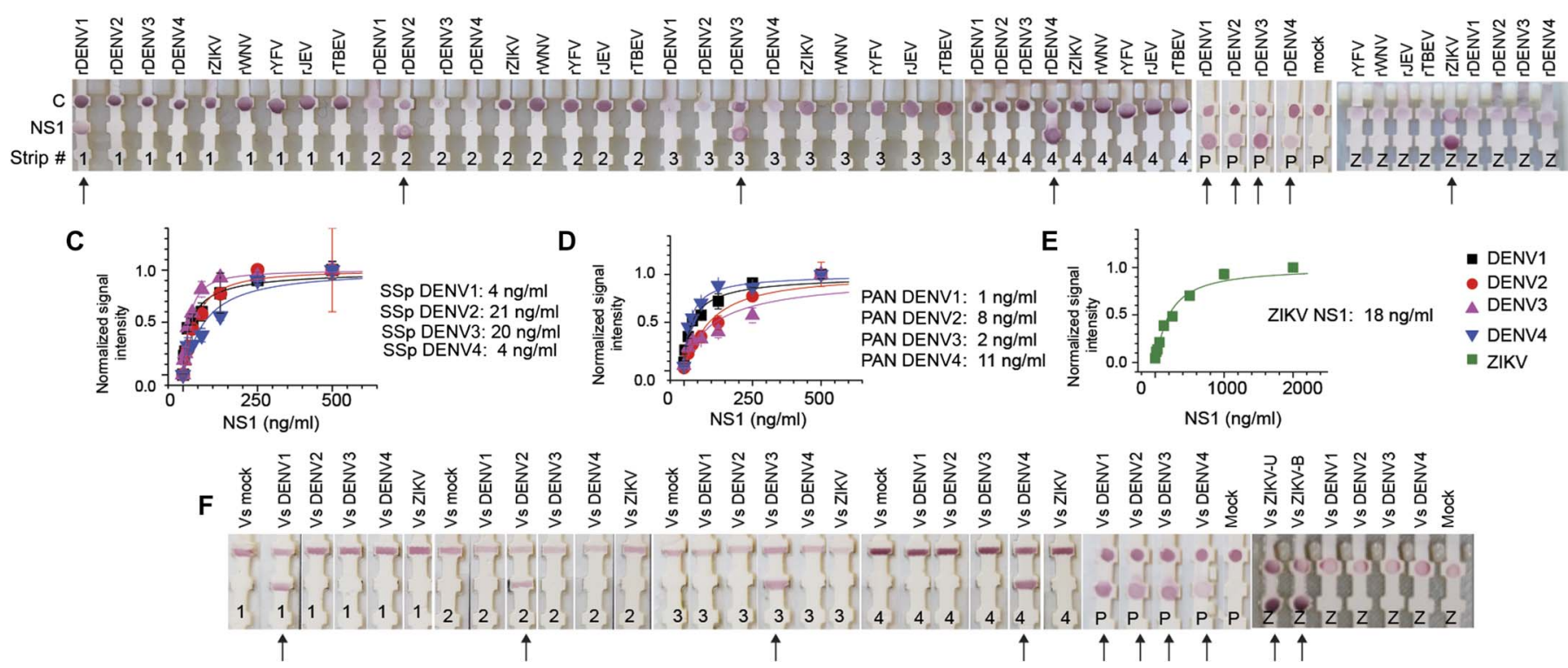

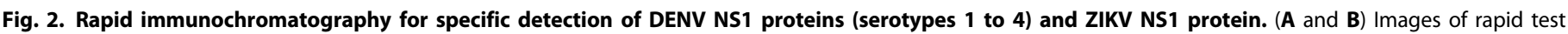

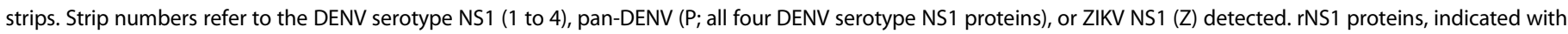

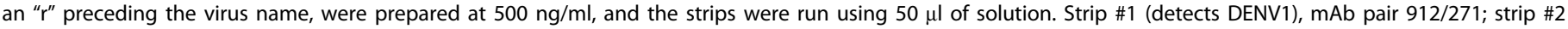

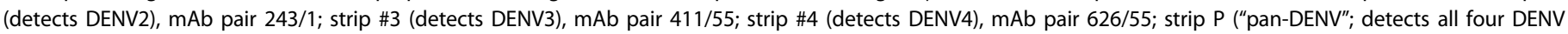

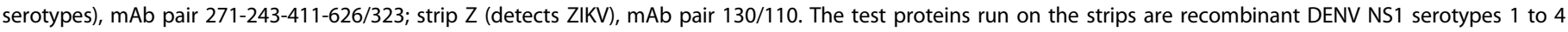

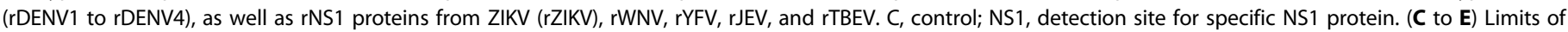

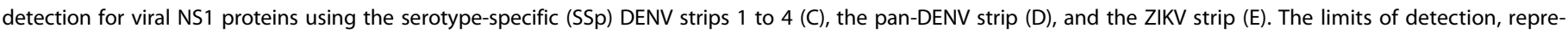

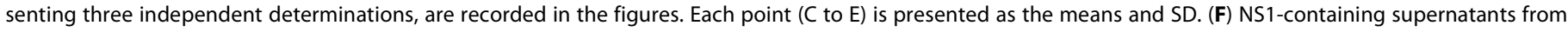

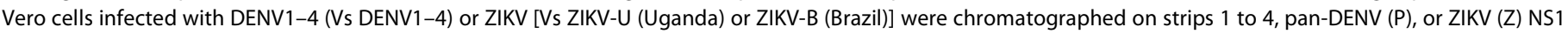

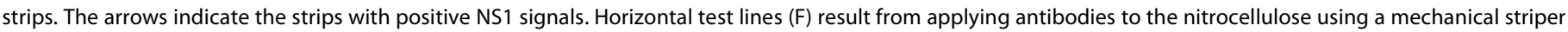
device; the circular dot signals result from applying antibodies to the nitrocellulose using a standard pipettor.

each of the antibody pairs, we chromatographed dipsticks 1 to 4 (detecting DENV1-4), pan-DENV (P, detecting all four DENV serotype NS1 proteins), or ZIKV (Z) using solutions of the following rNS1 proteins (500 ng/ml): DENV1-4, ZIKV, West Nile virus (WNV), yellow fever virus (YFV), tick-borne encephalitis virus (TBEV), and Japanese encephalitis virus (JEV). Figure 2A shows DENV1-4, and Fig. 2B shows pan-DENV and ZIKV strips. The results demonstrate that specific NS1 signals were observed for each of the DENV serotype NS1 proteins on the appropriate strip (Fig. 2, arrows). Strip 1 detected DENV1 rNS1 protein expressed by mammalian cells, with no detectable interaction with the other DENV serotype proteins or with other flavivirus rNS1 proteins. Similar results were observed with strip 2, where DENV2 NS1 was detected; also with strip 3, where DENV3 NS1 was detected; and with strip 4, where DENV4 NS1 was detected.

Dipstick "P" strips in Fig. 2B show the performance of the panDENV strip that detects all four DENV serotype NS1 proteins. The "Z" strips demonstrate the performance of the ZIKV strips. To prepare the pan-DENV nanoparticles, four mAbs $(271,243,411$, and 626) were covalently coupled as a mixture to gold nanoparticles, and $\mathrm{mAb} 323$ (Fig. 2B and table S4) was adsorbed to the nitrocellulose membrane. The data demonstrate that all four DENV serotype proteins were detected using the pan-DENV strip. Figure 2B (right) also shows the ZIKV strip detecting recombinant ZIKV NS1 protein ( $Z$ strips). These results indicate that the DENV and ZIKV strips are specific in detecting recombinant DENV serotype NS1 proteins or recombinant ZIKV NS1 protein without cross-reactivity interference from other flavivirus NS1 proteins.

The limits of detection for the pan-DENV, serotype-specific DENV, and ZIKV NS1 tests were defined by chromatographing dilutions of rNS1 proteins and then quantifying the signal intensities, normalizing them to the plateau maximum, and plotting sigmoidal data fits (Fig. 2, $\mathrm{C}$ to $\mathrm{E})$. The limits of detection were calculated as the NS1 concentrations intersecting a line representing a signal intensity point fivefold greater than the SD of the background signal. The results show that the limits of detection for the DENV1-4 NS1 proteins ranged from $\sim 4$ to $21 \mathrm{ng} / \mathrm{ml}$, whereas the range was 1 to $11 \mathrm{ng} / \mathrm{ml}$ for the pan-DENV strip. These limits of DENV NS1 detection are far below the reported concentrations of DENV NS1 protein in serum (up to $50 \mu \mathrm{g} / \mathrm{ml}$ ) (2). The limit of detection for the ZIKV strip was $18 \mathrm{ng} / \mathrm{ml}$. Serum NS1 concentrations in ZIKV-infected patients have not been reported. For comparison with current NS1 rapid tests, we chromatographed DENV NS1 proteins on DENV NS1 rapid tests from Standard Diagnostics (fig. S3). The results suggest that limits of detection for the DENV rapid tests that we developed (described in Fig. 2) are under $75 \mathrm{ng} / \mathrm{ml}$, whereas the limit of detection for the Standard Diagnostics tests is between 75 and $150 \mathrm{ng} / \mathrm{ml}$.

As a final validation step preceding patient sample analysis, the dipstick strips were tested in the laboratory by chromatographing native NS1 protein released by virus-infected Vero cells into cell culture supernatants (Fig. 2F). Although the rNS1 proteins used in Fig. 2 were 
expressed by eukaryotic cells to optimize antigen protein folding with secondary modifications such as glycosylations, testing the binding of native NS1 proteins released by virus-infected cells is a more robust proxy for analyzing clinical serum samples. Vero cells were infected individually with DENV serotypes 1 to 4 or with ZIKV (MR766 Uganda or contemporary Asian/American strains). Cell culture supernatants were collected and chromatographed on the dipsticks in a manner similar to Fig. 2A. The data in Fig. 2F show the recognition of test dipsticks 1 to 4 , as well as the pan-DENV strip, with each of the DENV1-4 Vero cell supernatants. These results are evidence that native NS1 protein expressed by DENV- or ZIKV-infected Vero cells yielded positive signals on the corresponding DENV1-4, pan-DENV, and ZIKV dipstick tests, without detectable cross-reactive binding (arrows).

As a comparison with current commercial DENV rapid tests, we chromatographed NS1-containing supernatants from ZIKV-infected Vero cells on Standard Diagnostics DENV rapid tests. In contrast to the lack of cross-reactivity observed in Fig. 2F, the Standard Diagnostics test showed cross-reactivity with the Vero cell supernatant (fig. S4, lanes 1 to 4). To extend these tests, we also chromatographed recombinant ZIKV NS1 protein on a Standard Diagnostics test. The results (fig. S4, lane 7) are consistent with the Vero cell supernatant results (lanes 1 to 4), confirming that the Standard Diagnostics test shows cross-reactive binding when ZIKV NS1 protein from ZIKV-infected Vero cells or recombinant ZIKV NS1 protein $(500 \mathrm{ng} / \mathrm{ml})$ was run. These results support a premise of this work that consideration of DENV/ZIKV NS1 cross-reactivity is essential in evaluating rapid diagnostics.

\section{Validating the immunochromatography tests using clinical serum samples}

We next turned to detecting ZIKV and DENV NS1 in a retrospective study of de-identified surveillance serum samples from human patients. Serum samples were tested in Brazil, Mexico, Colombia, Panama, Guatemala, and India (Fig. 3A), following approved human subjects use protocols. The sera were banked frozen samples representing blood that was drawn from febrile patients during routine care. Before using the rapid tests, the DENV and ZIKV serum samples were validated by reverse transcription polymerase chain reaction (RT-PCR), and positive tests were also evaluated using a laboratory-generated ELISA. The pan-DENV ELISA (Fig. 3B, left) was validated by testing supernatants of Vero cells infected with molecularly defined DENV or ZIKV isolates. As expected, the supernatants from DENV-infected Vero cells gave high signals in the DENV ELISA (lane 1), but no signal in the ZIKV ELISA (lane 6); ZIKV NS1 was detected from ZIKV-infected cell supernatant in the ZIKV ELISA (lane 7) but did not cross-react in the DENV ELISA (lane 2). Serum samples from uninfected patients were not recognized in the ELISAs (Fig. 3B, lanes 3 and 8). However, serum samples from DENV-infected patients yielded strong ELISA signals in the DENV ELISA (lane 4), but not in the ZIKV ELISA (lane 9). Conversely, sera from ZIKV-infected patients did not yield signal in the DENV ELISA (lane 5) but gave clear signals in the ZIKV ELISA (lane 10). These results strongly suggest that the validated ELISA detected DENV NS1 and ZIKV NS1 proteins without cross-reactivity interference. In the analysis of 25 ZIKV infections and 51 DENV infections, the serum NS1 concentrations were found to be lower in ZIKV-infected patients (lane 10) than in DENV-infected patients (lane 4). The concentrations of NS1, about $30 \mathrm{ng} / \mathrm{ml}$ (ZIKV) and $120 \mathrm{ng} / \mathrm{ml}$ (DENV), are consistent with other reports of low ZIKV viremia (16). The availabil- ity of the DENV and ZIKV ELISAs (Fig. 3B) allowed us to validate ZIKV serum samples for subsequent testing in the dipstick rapid tests.

Representative serotype-specific DENV detection using deidentified surveillance serum samples is shown in Fig. 3C. Each sample was tested using a panel consisting of five strips, with strips 1 to 4 recognizing DENV serotypes 1 to 4, respectively, and strip P recognizing all four serotype NS1 proteins (pan-DENV, confirming DENV infection). Although the specific serotype signals are visually apparent, to remove any subjectivity in reading and interpreting the results, we captured images of the test strips using a mobile phone camera and performed automated image recognition and processing using ImageJ to objectively quantify the data. Figure 3 (D to $G$ ) demonstrates that each of the specific serotype signal signals was statistically distinct from the other serotype signals $(P<0.001)$. A representative example of serum from a ZIKV-infected patient chromatographed on the ZIKV dipstick (Fig. 3C, right; ZIKA) shows that the serum sample $(Z)$ is positive, but serum from an uninfected patient $(\theta)$ is negative. Quantification in Fig. $3 \mathrm{H}$ also demonstrates that test signal intensities were distinct from uninfected patients.

To test for DENV/ZIKV NS1 cross-reactivity, we chromatographed supernatants from Vero cells infected with DENV4 on DENV serotypespecific dipsticks 1 to 4 , the pan-DENV dipstick (P), and the ZIKV NS1 dipstick (Z). Supernatant from Vero cells infected with DENV serotype 4 was detected, as expected, in strip 4 and the pan strip (P) (arrows); however, there was no detectable signal in the ZIKV strip (Fig. 3I). Comparable experiments were run with supernatants from cells infected with the other three DENV serotypes, and the expected results were observed (Fig. 3I). Alternatively, when supernatant from C6/36 insect cells that were infected with ZIKV (strain NR-50210, Human/2016/Panama) was tested, there was no signal in strips 1 to 4 and $\mathrm{P}$, but positive signal in strip $\mathrm{Z}$ was detected (Fig. 3J, arrow). ZIKV nucleic acid has been detected in the semen (17), and DENV NS1 has been detected in the urine $(18,19)$; however, the presence of ZIKV NS1 in the urine of infected patients has not been confirmed. We obtained three paired patient samples of acute PCR-positive ZIKV serum and urine and concentrated each fivefold using a centrifugal filter. The chromatography results show positive signals and detection of ZIKV NS1 in the serum samples (Fig. 3K, strips labeled "S" at top), but no signal in any of the urine samples (strips labeled " $U$ " at top). Quantifications in Fig. 3 (L and $\mathrm{M}$ ) indicate that the ZIKV and DENV NS1 antigen strips do not cross-react. Quantification of NS1 from five serum/urine pairs is shown in Fig. 4N. Overall, the results shown in Fig. 4 demonstrate serotype-specific DENV NS1 and ZIKV NS1 detection in clinical serum samples, without detectable crossreactivity interference.

\section{Rapid test detection window}

Rapid test NS1 detection was correlated with days after onset of symptoms when disease metadata were available. Because the DENV human serum samples used in this study were retrospective frozen surveillance samples, metadata were not available in all cases. Metadata from DENV samples from India and Colombia, as well as ZIKV samples from Guatemala and the Dominican Republic, included the fever onset day and fever duration. Normalized rapid test signals for DENV and ZIKV detection are shown in fig. S5 (A and B, respectively). The results suggest that the DENV rapid tests detected DENV NS1 at days 2 to 5 after onset of symptoms, in agreement with published results (6). The ZIKV test detected ZIKV NS1 at days 2 to 8 (fig. S5B); however, greater numbers of samples and controlled clinical studies will be 
A

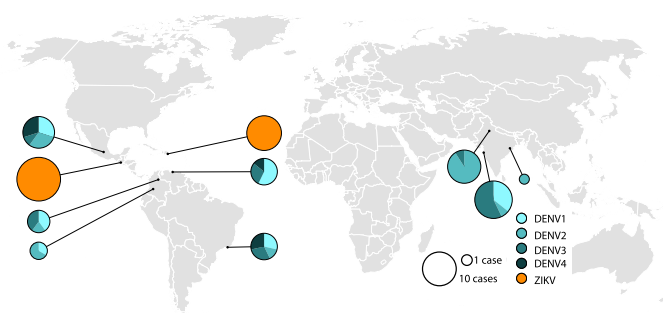

B

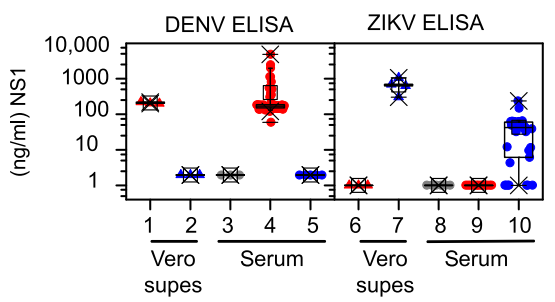

C

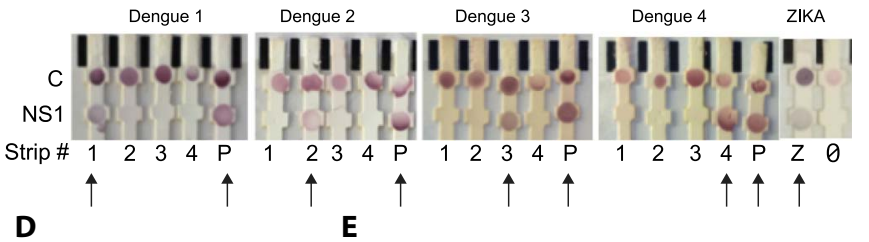

$\mathbf{E}$

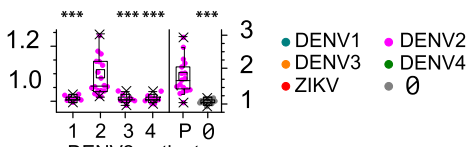

G

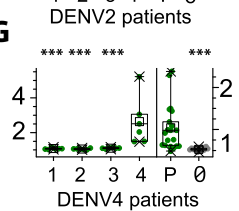

H

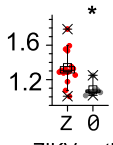

ZIKV patients

J ZIKA $\quad \mathbf{K}_{\text {ZIKA serum vs urine }}$

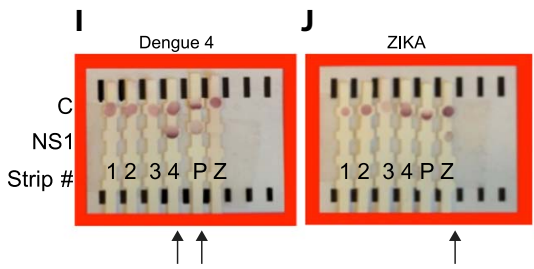

$\mathbf{L}$

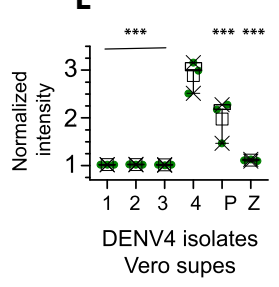

M

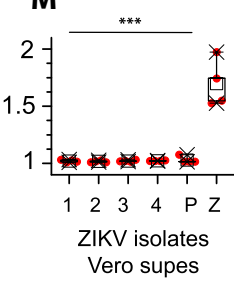

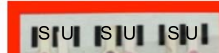

$\begin{array}{lllll}z & Z & Z & Z & Z\end{array}$

11111111

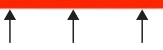

$\mathbf{N}$

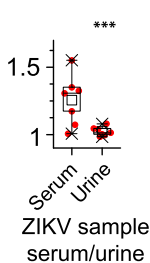

needed in future work to confirm the detection window. For comparison, RT-PCR-based virus detection has been reported for DENV (20), and also ZIKV, as measured in nonhuman primates (21).

Test data were analyzed further to define sensitivity (identifying true positives; individuals who have disease) and specificity (true negatives; individuals without disease). We evaluated sensitivity and

Fig. 3. Applying the rapid test to analyze human patient sera. (A) Map showing the endemic virus regions where the rapid tests were deployed to analyze patient serum samples. The areas of the circles correlate with the numbers of samples analyzed. The blue colors, faint to dark, represent DENV1-4. ZIKV is indicated in orange color. (B) ELISA results showing the amounts of DENV NS1 (left) and ZIKV NS1 (right) found in patient serum and supernatants from infected cell cultures. Lanes 1 and 6 are supernatants from Vero cells infected with DENV; lanes 2 and 7 are supernatants from Vero cells infected with ZIKV. Lanes 3 and 8 are PCR-negative sera; lanes 4 and 9 are sera from PCR-positive DENV patients. Lanes 5 and 10 are sera from PCR-positive ZIKV patients. (C) Images of rapid test analysis of DENV NS1 serotypes 1 to 4 and ZIKV NS1 on serotype-specific strips 1 to 4 , as well as pan-DENV (P) and ZIKV (Z); the upward arrows mark positive tests, and $\theta$ is serum from an uninfected patient. (D to $\mathbf{G})$ Quantification of rapid test results. Dipstick tests were run with PCR-confirmed DENV sera or ELISA-validated ZIKV serum $(C)$, and the resulting signals were quantified and expressed as box plots. Statistical significance, based on one-way analysis of variance (ANOVA), is indicated as ${ }^{* *} P<0.001$. (H) Statistical significance, based on an unpaired $t$ test, is presented as ${ }^{*} P<0.05$. In the box-and-whisker plots, the black $\times$ represents the maximum and minimum measured normalized intensity values, whereas the small square box ( $\square$ ) represents the mean value, and the larger box represents the 25 to $75 \%$ range of the data. Individual colored points represented individual patient samples measured. (I and $\mathbf{J}$ ) Images of rapid tests showing that DENV and ZIKV NS1 tests do not cross-react. (I) Supernatants from Vero cells infected with DENV4 were chromatographed on DENV serotype strips 1 to 4 on the pan-DENV strip (P) and on the ZIKV NS1 strip (Z). (J) Supernatants from Vero cells infected with ZIKV were chromatographed on DENV serotype strips 1 to 4 , on the panDENV strip (P), and on the ZIKV NS1 strip (Z). (K) Images of rapid tests showing ZIKV NS1 are detected in serum samples concentrated five times, but ZIKV NS1 is not detected in concentrated urine. Three sets of paired serum and urine samples were concentrated five times by filter centrifugation and chromatographed on the ZIKV dipsticks. S, serum; U, urine. (I to K) The red boxes and vertical black lines serve as fiducial markers for image recognition and processing. Upward arrows indicate positive tests using the serum samples. (L to $\mathbf{N}$ ) Quantification of NS1 protein in supernatants of Vero cells infected separately with three DENV4 patient isolates $(\mathrm{L})$ or three ZIKV patient isolates $(\mathrm{M})$ or five paired serum/urine patient samples (N). ( $L$ to N) One-way ANOVA was used to calculate statistical significance of the dengue and Zika tests: ${ }^{* *} P<0.001$. In the box-and-whisker plots, the black $x$ represents the maximum and minimum measured normalized intensity values, whereas the black box ( $\square$ ) represents the mean value, and the larger box represents the 25 to $75 \%$ range of the data. Individual colored points represented individual patient samples measured.

specificity as a function of an intensity cutoff value, above which a test was scored positive and below which a test was considered negative. Receiver Operating Characteristic (ROC) curves illustrate the performance of the rapid tests as a function of the discrimination threshold, plotted as sensitivity versus 1 - specificity in Fig. $4 \mathrm{~A}$; numerical values are shown in Fig. 4B. The areas under the ROC curves are a proxy of test performance, where 1 represents a perfect test, and 0.5 represents a random predictor. We measured areas of $0.88,0.96,1,0.98$, 0.95 , and 0.82 for DENV1, DENV2, DENV3, DENV4, pan-DENV, and ZIKV, respectively. The calculated optimal cutoff values were 1.14, 1.18, $1.12,1.37,1.9$, and 1.08 for DENV1, DENV2, DENV3, DENV4, panDENV, and ZIKV, respectively, using a bootstrap technique with 1000 iterations. Sensitivity is defined as the number of measured positives divided by the total confirmed positives, and specificity is defined as the measured negatives divided by the total of confirmed negatives. Using the optimal cutoff value, the test sensitivity and specificity were as follows: $0.76(13 / 17)$ and 0.89 (33/37) for the DENV1 test, 0.89 (8/9) and $0.98(44 / 45)$ for the DENV2 test, $1(16 / 16)$ and $1(39 / 39)$ for the 

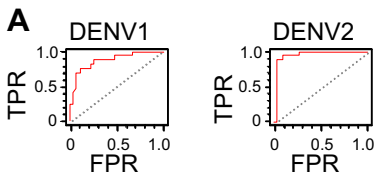

B
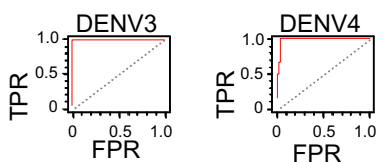

\begin{tabular}{|c|c|}
\hline & DEN \\
\hline $\begin{array}{c}\text { Area under } \\
\text { the curve }\end{array}$ & 0.88 \\
\hline $\mathbf{9 5 \%}$ Conf. int. & 0.74 \\
\hline Cutoff & 1. \\
\hline Sensitivity & 0.76 \\
\hline Specificity & 0.89 \\
\hline N positive & 1 \\
\hline N negative & 37 \\
\hline
\end{tabular}
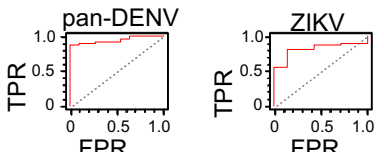

$\mathrm{N}$ negative

FPR

Fig. 4. ROC analysis, sensitivity/specificity analysis, and $95 \%$ confidence intervals of the dengue and Zika tests. (A) ROC curve analysis of the patient sample data collected for DENV1-4, pan-DENV, and ZIKV. TPR, truepositive rate (sensitivity); FPR, false-positive rate (1 - specificity). (B) Table listing numerical values of the sensitivity and specificity results. AUC, area under the curve; Conf. int., confidence interval.

DENV3 test, 1 (6/6) and $0.96(44 / 46)$ for the DENV4 test, $0.88(51 / 58)$ and $1(11 / 11)$ for the pan-DENV test, and finally, $0.81(25 / 31)$ and $0.86(6 / 7)$ for the ZIKV test.

\section{DISCUSSION}

Rapid diagnostics provide critical information that informs patient care and assesses patient risks, generally within 1 hour after sample collection. Despite urgent needs, efficacious and inexpensive rapid tests are not available for many infectious diseases including arboviruses (mosquitoand tick-transmitted viruses). Viral antigen-based tests provide important patient benefits in geographic areas where molecular tests such as RT-PCR are not available, have very slow turnaround times, or are prohibitively expensive. Rapid tests have further applications in the developed world, where immediate results may be required in the context of febrile travelers at airports or for military personnel serving in endemic areas. Accurate pathogen identification is essential, a goal that is complicated when distinguishing closely related cocirculating viruses such as DENV and ZIKV, which have markedly different risk profiles. The similarity of clinical symptoms is a compounding problem in many cases and also a justification for improved diagnostics that provide accurate data on which to base clinical decisions. DENV, ZIKV, and chikungunya (an alphavirus) infections exhibit common symptoms including varying degrees of fever, headache, myalgia, arthralgia, nausea, and rash, with hemorrhagic fevers as outcomes of severe infections in about $1 \%$ of infected individuals (22). However, the ZIKV outbreak stunned the virology and medical communities with a distinct set of risks and severe outcomes not previously associated with flavivirus infections, including fetal microcephaly and Guillain-Barré syndrome. Because DENV and ZIKV have major impact on economically challenged countries in the tropics, our approach has been guided by the World Health Organization acronym "ASSURED” to describe the ideal characteristics of a diagnostic test that can be used at all levels of the health care system: affordable, sensitive, specific, user-friendly, rapid, equipment-free, and delivered to those who need them $(23,24)$.

This report describes DENV rapid tests that specifically detect the viral NS1 protein to identify and distinguish the four DENV serotypes without observed cross-reaction with ZIKV. Despite the high percentages of homology and identity among the flavivirus NS1 proteins (table S2), our screening strategy (fig. S1 and table S1) identified antibody pairs that detected and distinguished the four DENV serotype NS1 proteins, as well as ZIKV NS1 (recombinant and expressed by virus-infected cells), without detectable cross-reactive interactions. These results are important for several reasons. First, we have identified antibody pairs that distinguish closely related NS1 proteins and applied them to create rapid tests. Success in detecting/distinguishing the individual serotypes was dependent on (i) immunizing groups of mice with the native dimer/hexamer forms of rNS1 proteins that were expressed by eukaryotic cells; (ii) initiating hybridoma screening against multiple NS1 proteins in parallel, enabling us to use ELISA and flow cytometry methods to sort and select from the most diverse pool; and (iii) performing systematic unbiased pairwise screening of the selected clones to identify specific antibody pairs to recognize specific serotypes (table S3). Similar approaches may be used to identify and distinguish other closely related proteins. Second, epidemiological surveillance is a second area of importance for serotype-specific detection and is a critical component of patient care and public health preparedness $(16,25,26)$. The serotype-specific tests described here could be used in inexpensive patient screening during routine medical care to identify not only incoming serotypes but also patients in endemic areas who may have asymptomatic infections. The introduction of an additional DENV serotype into a region may cause outbreaks with severe hemorrhagic fever presentation because the individual serotype infections do not provide long-lasting cross-protection, and heterologous infections increase the hemorrhagic fever outcome of dengue. Therefore, we propose that rapid testing may diminish the impact of emerging epidemics by enabling early detection of outbreaks. Third, serotype-specific detection has implications for DENV vaccine development because current vaccine candidates may not show equal protection across the serotypes (27), and knowledge of circulating serotypes can inform trials and outcomes. Finally, the approaches for rapid tests described here represent a platform that can be applied toward detecting the future emergence of new pathogens. Kuno et al. (28) report about 70 viruses in the genus Flaviviridae alone, and the methods described here can be applied toward detecting and distinguishing other potentially emergent flaviviruses while minimizing cross-reactivity.

In addition to detecting and distinguishing DENV serotypes, we report here a rapid test for ZIKV NS1. Laboratory testing demonstrated that the ZIKV NS1 test does not cross-react with DENV NS1 (Figs. 2 and 3 , and table S4). Detection without cross-reaction was also confirmed using patient samples (Fig. 4). Detecting ZIKV NS1 in patient samples was more challenging than detecting DENV NS1 because ZIKV NS1 concentrations were about 10 -fold lower than DENV patient NS1 (Fig. 3). We addressed this by concentrating the sera using a centrifugal filter (a 5-min centrifugation at 10,000g). As shown in Fig. 4, ZIKV NS1 was detectable in samples that were concentrated fivefold; that is, to $30 \mu \mathrm{l}$ from a starting volume of $150 \mu \mathrm{l}$. Although urinary DENV NS1 has been reported $(3,29)$, ZIKV NS1 was not detected here in $5 \times$ concentrated urine samples, either by ELISA or rapid tests. We cannot rule out the possibility that urinary ZIKV NS1 is present at concentrations that are below detection by our assays.

The use of image processing and computer software to analyze rapid tests has been described previously in nonclinical analysis $(30,31)$. 
Mobile image capturing and image processing ensure objective and quantified data under varied use conditions and signal intensities and generate standardized data that can be shared and compared on a global basis. Tests showed both very high signal intensities, especially with DENV detection, and low signal intensities, often with ZIKV NS1 (Figs. 3 and 4). However, low signal intensity is not restricted to ZIKV because DENV NS1 signals can also be very low immediately after the onset of disease symptoms (days 0 to 1 ) and also about 6 to 7 days after onset of symptoms when the virus is being cleared (32). Although the human eye is extremely sensitive, machine vision offers improved performance for quantitative measurement (33). Andries et al. (34) reported wide differences in sensitivity and specificity when different people at different sites evaluated the same diagnostic. These observations suggest that training and oversight are required in the use of rapid tests (34). Image processing and data quantification, as described here, avoid errors in user-based interpretation. The approach is independent of computation capacity of the phone because the computation is performed "in the cloud". Immediate Internet connectivity is not required in field applications of the tests because our store-and-forward software enables the coded de-identified image data to be stored on the phone and uploaded or transferred at later times for analysis. The ubiquity of mobile phones allows test users to obtain an objective analysis based on a common algorithm shared across a global network.

The DENV and ZIKV tests described here compare very well with rapid tests described previously $(4,6,7,9,34-36)$, with the added crucial benefit of avoiding detectable cross-reactivity between ZIKV and DENV and among ZIKV/DENV and a number of other flavivirus NS1 proteins. Nonetheless, the current DENV and ZIKV tests described here have some limitations, and opportunities for improvement remain. Using whole blood rather than serum would simplify use as a point-ofcare diagnostic in the clinic. The tests reported here were performed using serum because the clinical samples available for retrospective analysis in the endemic areas were frozen serum samples that were validated for virus infection by RNA extraction and nucleic acid amplification and/or by ELISA for the NS1 protein. For whole-blood analysis, we have developed and successfully tested lateral flow chromatography devices that incorporate our antibodies and use a specialized sample pad paper that removes red blood cells; therefore, we do not anticipate the use of blood as an obstacle for future versions of the rapid tests. The sensitivity and specificity of the ZIKV tests ranged from 0.7 to 1.0. The sample availability for Zika testing was very limited because of the fact that ZIKV is an epidemic that only recently emerged. Further ZIKV testing and device optimization will be possible as the availability of patient samples improves. The current state of research is that it is very difficult to obtain validated acute-phase ZIKV patient samples with complete metadata, a challenge that is also reflected in single sample data points reported recently from the ZIKV outbreak in Florida, USA (37). Viral NS1 concentrations vary in individual patients and with time after infection (38). ZIKV test performance was improved by concentrating serum samples. Although centrifugation often requires instrumentation and power, in contradiction with the ASSURED paradigm, unpowered "whirligig" centrifugation devices have been described recently (39), which could concentrate samples or separate serum while meeting the ASSURED criterion of "equipment-free." The concentration step was not needed to detect serum ZIKV NS1 using the laboratorymade ELISA with $150 \mu \mathrm{l}$ of serum. A rapid test that could accommodate a $150-\mu$ l sample volume (currently limited by the wick absorption) may permit greater sensitivity, although it would likely be necessary to increase the number of conjugated nanoparticles used in the test.
Device cost and detection during secondary infections are important issues to consider. The current cost for each strip (Figs. 3 and 4) is nearly $\$ 5.00$, which is due primarily to using commercial gold nanoparticles, coupled with small-scale antibody production. The cost of the nanoparticles can be decreased by 1000 -fold by using laboratory-made nanoparticles that have excellent performance (fig. S6); antibody production scale-up will further decrease costs. We considered the possibility that the efficacy of our rapid tests for detecting NS1 might be compromised in secondary dengue infections, where circulating anti-NS1 antibodies could bind NS1 to form immunocomplexes that would shield NS1 from recognition by the rapid test antibodies. Using our DENV diagnostic, we tested a number of confirmed secondary dengue infection samples that had been confirmed by serological rapid tests and hemagglutination tests that established relative IgG/IgM concentrations. We successfully detected DENV NS1 protein in secondary infections (fig. S7), consistent with a recent report (40). We speculate that detecting NS1 in secondary dengue infections might be more feasible than detecting envelope (E) protein because the interfering anti-E antibodies are present in larger quantities in the serum and in higher amounts compared with the anti-NS1 antibodies. Therefore, a test based on E detection may be blocked via competition between serum polyclonal antibodies and the mAbs used in the nanoparticle.

The rapid NS1 antigen test described here is effective only when analyzing samples collected during the acute phase of the virus infection, when flavivirus RNA and NS1 are detectable, before virus clearing by the immune system. Serological tests for anti-envelope protein IgG/IgM or anti-NS1 protein IgG/IgM are useful for evaluating patients' postacute phase. Several companies are marketing DENV and ZIKV IgG/ IgM tests, although in many cases, their cross-reactivity has not been evaluated. A new approach, reported recently, shows high sensitivity and specificity for ZIKV immunoglobulins (41). We propose that optimal patient care will be provided through the use of several diagnostic approaches that are applicable for a range of clinical needs. Rapid immunochromatography tests are well suited for fast turnaround times without need for specialized reagents, equipment, or trained personnel. Rapid tests are generally low cost, can often be transported without refrigeration, and can be used in austere environments. Nucleic acid amplification methods are highly specific, with low limits of detection as compared to immunochromatography strips; however, disadvantages include requirements for equipment powered by electricity/batteries, specialized reagents, and a cold chain for maintaining enzyme activity. Synthetic biology approaches (42) may offer a hybrid approach wherein the simplicity of paper diagnostics can be combined with isothermal nucleic acid amplification. There is great need for all of these technologies to improve the time to diagnosis in patients infected by pathogens worldwide.

In summary, we identified here $\mathrm{mAb}$ pairs and developed rapid tests that detect and distinguish DENV NS1 antigen for serotypes 1 to 4, pandengue, and ZIKV NS1 without detectable cross-reactivity. We report the limits of detection of each antibody pair, demonstrating that DENV NS1 can be detected using serotype-specific tests and pan-DENV tests in the range from 1 to $20 \mathrm{ng} / \mathrm{ml}$ and ZIKV NS1 detection at about $20 \mathrm{ng} / \mathrm{ml}$. The strips have been validated using $\mathrm{rNS} 1$ protein, NS1 present in the supernatants of virus-infected Vero cells or C6/36 cells, and NS1 protein present in clinical serum samples from several geographic areas in the Americas and India. The use of a mobile phone camera linked to Image J analysis of the rapid tests permitted objective analysis of the test data. Together, we demonstrate that we have developed and characterized NS1 antigen tests capable of distinguishing the DENV serotypes 
and detecting ZIKV and DENV infections without cross-reactivity, in anticipation of broader clinical applications.

\section{MATERIALS AND METHODS Study design}

The overall objective of the study was to develop and validate a platform approach for producing low-cost paperfluidic rapid antigen tests to detect viruses while minimizing cross-reactivity and false-positive signals. The platform approach centers on screening mAbs against panels of multiple flavivirus NS1 antigens to assess binding specificity, relative binding affinity, and cross-reactivity. DENV and ZIKV are the focus of this report; however, the approach can be used with any of the estimated 70 flavivirus NS1 proteins. mAb linear epitope-binding sites were defined, and the antibodies were tested pairwise in dipstick tests to identify those that yielded specific NS1 binding without detectable cross-reactivity. Limits of detection were defined using rNS1 proteins. The dipstick tests were validated in endemic areas using de-identified serum samples that were collected from febrile patients. Most of the testing was performed on-site in Central America, South America, and India. The serum samples were validated using nucleic acid amplification methods and NS1 ELISAs to generate validated test panels. To remove subjectivity from the test result readings, mobile phone cameras were used to capture test strip images, and the test signals were quantified using ImageJ. These quantitative data were then used to generate test sensitivity and specificity values using ROC analysis.

\section{mAb production strategy}

DENV and ZIKV anti-NS1 mAbs were produced in mice under contract (Covance Inc.), following an approved animal care protocol. $\mathrm{BALB} / \mathrm{c}$ mice were immunized with purified recombinant DENV NS1 or ZIKV NS1 proteins that were expressed in mammalian cells (Native Antigen Co.) to facilitate protein folding and posttranslational modifications. From each of the immunized groups, one seroconverted animal with high titers of antibodies recognizing a pool of the NS1 DENV antigens was used for cell fusion to generate hybridomas. Supernatants from cloned hybridomas were tested by ELISA to generate "fold over background" values that gave us an approximate idea of serotype specificity or DENV/ZIKV specificity (see table S4). From the $\sim 200$ DENV hybridomas and 100 ZIKV hybridomas identified in the initial screening (table S1), supernatants from 30 DENV clones and 16 ZIKV clones were used to stain virus-infected Vero cells, followed by flow cytometric analysis. This step was included to ensure that the selected hybridomas produced antibodies that recognized native NS1 protein expressed by virus-infected cells. About 15 anti-DENV NS1 hybridomas and 16 anti-ZIKV NS1 hybridomas were expanded and grown in low IgG serum (Invitrogen) containing hybridoma cloning supplement (Roche). The expressed mAbs were isotyped and purified by affinity chromatography on a protein L or A matrix (GE Healthcare). Purified antibodies were concentrated and buffer-exchanged into phosphate-buffered saline (PBS). Eleven purified DENV antibodies were then tested pairwise in immunochromatography tests, as were $10 \mathrm{ZIKV}$ antibodies to identify pairs that exhibited high differential NS1 binding and low nonspecific background interactions.

Anti-ZIKV mAbs were generated using a modified immunization and $B$ cell harvest protocol. Both a rapid lymph node approach (B cell isolation from lymph nodes and cell fusion at 23 days after immunization) and the more traditional spleen B cell hybridoma method (B cell isolation from $\mathrm{B}$ cells and cell fusion at 105 days after immunization) were used. To select antibodies with minimal potential for cross-reactive DENV NS1 recognition, we also applied an enhanced screening approach. About 100 anti-ZIKV NS1 hybridoma supernatants were screened by indirect ELISA against not only ZIKV NS1 protein but also DENV1-4 NS1 proteins, as well as a panel of flavivirus NS1 proteins including WNV, Usutu virus, TBEV, and JEV. The antibody distribution data (fig. S2, right) demonstrate that the proportion of ZIKV NS1specific antibodies was higher for the lymph node fusion approach than the traditional spleen cell fusion method. From the starting pool of about 100 anti-ZIKV mAbs, 16 anti-ZIKV mAbs were selected for step 2 by flow cytometric analysis.

\section{Antibody conjugation to nanoparticles}

Forty-nanometer gold nanoparticles were purchased from Innova Biosciences. The serotype-specific DENV nanoparticle-antibody conjugates and the ZIKV nanoparticle-antibody conjugates were prepared according to the manufacturer's instructions. For single antibody conjugations, the antibody was first diluted to $0.1 \mathrm{mg} / \mathrm{ml}$ in the supplied dilution buffer. Next, $12 \mu \mathrm{l}$ of diluted antibody was mixed with $42 \mu \mathrm{l}$ of reaction buffer. Forty-five microliters of the mix was then used to suspend the lyophilized gold nanoparticles [particles with an optical density at $\left.20 \mathrm{~nm}\left(\mathrm{OD}_{20}\right)\right]$. The antibody-nanoparticle mix was incubated for $10 \mathrm{~min}$ at room temperature, followed by the addition of $5 \mu \mathrm{l}$ of proprietary quencher solution to stop the coupling reaction. After adding the quencher solution, $100 \mu \mathrm{l}$ of $1 \%$ Tween 20 in PBS and $50 \mu \mathrm{l}$ of $50 \%$ sucrose in water were added to the conjugates before use in immunochromatography. The pan-DENV nanoparticles used for detecting all four DENV serotypes were conjugated using a mixture of four different antibodies. These particles were created by preparing $12 \mu \mathrm{l}$ of solution containing each of the four antibodies (MA724.271, MA7732.243.108, MA724.411, and MA726.626) at a final concentration of $0.1 \mathrm{mg} / \mathrm{ml}$ (final total antibody concentration, $0.4 \mathrm{mg} / \mathrm{ml}$ ). Twelve microliters of the resulting antibody mixture was then mixed with $42 \mu \mathrm{l}$ of reaction buffer and processed according to the same protocol as the other conjugates.

\section{Antibody application to nitrocellulose membranes}

Nitrocellulose membrane (HF18002XSS, EMD Millipore) was cut into strips using a laser cutter (Universal Laser Systems; model VLS2.30; $30 \mathrm{~W}$ ) at $30 \%$ power and $90 \%$ speed. The strip pattern was designed in Adobe Illustrator. Strips were attached to a wick (GB003, Gel Blot Paper) with adhesive paper (MIBA-010 Backing Card, 0.51-mm thickness; DCN Diagnostics). For the positive control area, $0.33 \mu \mathrm{l}$ of anti-mouse Fc antibody ( $1 \mathrm{mg} / \mathrm{ml}$; AQ127, EMD Millipore) was spotted on the control line. The anti-NS1 capture line on the nitrocellulose was generated by pipetting $0.33 \mu \mathrm{l}$ of anti-DENV NS1 antibody $(4 \mathrm{mg} / \mathrm{ml})$ at the NS1 test area. Strips were air-dried and stored in a desiccator at room temperature before use.

\section{Immunochromatography}

Each immunochromatography strip was run in a separate microcentrifuge tube, and groups of tubes/strips were run together. The rapid test solution contained (i) $30 \mu \mathrm{l}$ of human acute DENV/ZIKV/uninfected serum sample, (ii) $30 \mu \mathrm{l}$ of fetal calf serum, (iii) $5 \mu$ l of proprietary quencher solution (Innova Inc.), and (iv) $16 \mu \mathrm{l}$ of conjugated gold nanoparticle mix (see antibody conjugation to nanoparticles). The run time varied with the sample and humidity; it was always less than 1 hour and sometimes as short as $15 \mathrm{~min}$. The strips were left to dry and then imaged for quantitative signal analysis. 


\section{ELISA for the detection and quantification of circulating ZIKV and DENV NS1}

Ninety six-well plates (flat bottom high binding, CoStar; cat. no. 3590) were coated with $100 \mu \mathrm{l}$ per well of optimized doses of mAb 110 diluted in $1 \times$ PBS (ref. 10010-023, Gibco) and incubated overnight at room temperature. After blocking with 5\% nonfat dry milk (cat. no. sc-2325, Santa Cruz Biotechnology) and 0.05\% Tween 20 (cat. no. p-1379, Sigma-Aldrich) in $1 \times$ PBS ( $5 \%$ blotto) for 1 hour, serial dilutions of rNS1 proteins from ZIKV, DENV, YFV, WNV, JEV, TBEV (all starting at $2000 \mathrm{ng} / \mathrm{ml}$ in $2.5 \%$ blotto), or undiluted serum/plasma samples or supernatant of ZIKV- or DENV-infected Vero cells were added and incubated for 2 hours at room temperature. To detect ZIKV NS1, the plates were washed three times with $0.1 \%$ Tween 20 in $1 \times$ PBS (wash buffer), followed by the addition of $100 \mu \mathrm{l}$ per well of optimized doses of biotin-labeled mAb 130 diluted in $2.5 \%$ blotto, followed by incubation for 1 hour at room temperature. After another washing step, $100 \mu \mathrm{l}$ per well of peroxidase-labeled streptavidin $(1 \mu \mathrm{g} / \mathrm{ml}$; Thermo Fisher Scientific, cat. no. 21130) was added, and the plates were incubated for 1 hour at room temperature. Finally, the plates were developed with tetramethylbenzidine single solution (cat. no. 002023, Life Technologies), and the reaction stopped with $50 \mu \mathrm{l}$ per well of $2 \mathrm{M}$ sulfuric acid (cat. no. 8315-32, Ricca Chemical Company). A TriStar LB 941 spectrophotometer (Berthold Technologies) was used to read the plates at a wavelength of $450 \mathrm{~nm}$. In the quantitative ELISA for the detection of DENV NS1, a protocol similar to ZIKV NS1 ELISA was followed, using the anti-DENV NS1 mAb 323 as capture antibody and the biotin-labeled $\mathrm{mAb} 323$ as detection antibody. A standard curve was generated with addition of mixed rNS1 protein from DENV1-4 (Native Antigen Co.), starting at $2000 \mathrm{ng} / \mathrm{ml}$. The samples were diluted $1 / 100$ in $2.5 \%$ blotto and run in duplicate. The mean $\mathrm{OD}_{450}$ of the negative controls (samples with previously known undetectable NS1) were 0.12 and 0.15 for ZIKV and DENV NS1 ELISA, respectively. Wells where only $2.5 \%$ blotto were added had similar $\mathrm{OD}_{450}$. The limit of detection of the assays was taken as the concentration of ZIKV and DENV rNS1 proteins with at least twofold higher $\mathrm{OD}_{450}$ than the background (samples with undetectable NS1). When analyzing clinical samples, the correlation coefficients of the standard curves of recombinant ZIKV and DENV NS1 were $\geq 94 \%$, and the duplicate variability was $<10 \%$. Calculating the concentration of ZIKV NS1 was performed by interpolation of the $\mathrm{OD}_{450}$ of the samples to the standard curve using four-parameter logistic regression with GraphPad Prism software version 7.0.

\section{Patient serum samples}

Each of the collaborating laboratories contributed a panel sample size of 6 to 20 de-identified retrospective serum samples. Massachusetts Institute of Technology (MIT) Institutional Review Board (IRB) and local IRBs approved the protocols for purposes of pathogen identification. Virus identity in human serum samples was confirmed by nucleic acid amplification (RT-PCR). Dengue infections were confirmed using Lanciotti primers (43). The following primers and probe sequences for ZIKV were provided by U.S. Centers for Disease Control and Prevention (CDC), Puerto Rico: 5' -CCGCTGCCCAACACAAG-3' (1086f ZIKV), 5'-CCACTAACGTTCTTTTGCAGACAT$3^{\prime}$ (1162 ZIKV), and 5'-FAMAGCCTACCTTGACAAGCAGTCAGACACTCAA-3' (1107-FAM). The serum panels used for the retrospective rapid test analysis of DENV and ZIKV acute sera were stored at each of the collaborating laboratories at $-80^{\circ} \mathrm{C}$ until use. To use the serum samples in immunochromatography, we first passed the serum through a Spin-X Centrifuge tube filter with a $0.2-\mu \mathrm{m}$ nylon filter (cat. no. 8169,
CoStar) to remove any cryo-aggregates. For ZIKV detection, $150 \mu \mathrm{l}$ of cleared filtered serum was then concentrated five times using a centrifugal filter (cat. no. UFC503096, Amicon Ultra; $0.5 \mathrm{ml}, 30 \mathrm{~K}$ ) that was centrifuged for $10 \mathrm{~min}$ at 13,000 rpm using an Eppendorf tube centrifuge. If sufficient volume of the serum sample was available $(100 \mu \mathrm{l})$, it was analyzed using the laboratory ELISA as described above. A sample size of $30 \mu \mathrm{l}$ of DENV serum, or $30 \mu \mathrm{l}$ of $5 \times$ concentrated (from $150 \mu \mathrm{l}$ ) ZIKV sample serum or urine, was used for the rapid tests.

\section{Image analysis}

Rapid test results were analyzed using image processing software to machine-read and quantify test results. After completion of the test run, the strips were taped to a sheet of paper with a printed red box outline that was slightly larger than the strip length. Within the red box, short black vertical lines served as fiducial markers for image processing, separating the strips and identifying top/bottom. The image of the strip inside the red box was captured with a mobile phone camera and analyzed using an ImageJ macro to quantify test results. ImageJ is used as a public domain image analysis software. The ImageJ macro provided instructions to localize and scan the positive control area and test area in each test. Briefly, a Workflow (DeskConnect Inc.) script was written to run on the mobile phone, with instructions to Global Positioning Systemlocalize where the image was captured, followed by uploading to Dropbox. A Python script running on a desk computer was activated upon Dropbox sync, calling ImageJ to perform the image analysis on the uploaded file. An Image J macro identified the red square and black line fiducial markers, followed by drawing a rectangle in between the black markers, on the strips. ImageJ quantified the signal at the test area and blank/background area and generated a normalized intensity, calculated by dividing the maximum intensity value at the test band by the average value of a blank area in each test strip. Test data were stored and used later for sensitivity/ specificity and ROC curve analysis.

\section{Limits of detection}

Limits of detection for each of the antibody pairs were measured using rNS1 proteins antigens. Antigen solutions were diluted serially and chromatographed on DENV1-4 dipsticks, the pan-DENV dipstick (P), or the ZIKV dipstick (Z). The signal intensities were quantified (ImageJ), normalized by the intensity at the highest concentration, plotted against antigen concentration, and fit to a sigmoidal curve. The limit of detection was calculated from the sigmoidal curve fit as the NS1 concentration found at the intersection with a line representing the value of 5 times the SD of background signal intensity. Background was determined by analyzing triplicate strips in nitrocellulose areas outside of the test and positive control regions.

\section{NS1 detection in secondary dengue infections}

To define primary and secondary dengue infection samples, we tested paired acute/convalescent serum samples from a Venezuelan cohort $(44,45)$ using a standard hemagglutination assay $(4,46)$ where virus particles bind to sialic acid residues on the surface of red blood cells, causing the red blood cells to agglutinate. Serum dilutions are added to the assay, and antivirus antibodies present in serum bind to virus particles and thereby inhibit the agglutination. The dilution factor marking the interface of hemagglutination inhibition/no inhibition is a measure of antivirus (specifically anti-envelope protein) antibodies. The World Health Organization has defined primary dengue infections as those with hemagglutination inhibition antibody titers equal to or less than 1:640, whereas secondary dengue infections have been 
defined as those with titers of equal to or greater than 1:1280. For the work reported here, diluted patient serum samples from dengue-infected patients were mixed with red blood cells, and agglutination status was scored. Samples with antibody titers $>1280$ were defined as secondary infections and analyzed for NS1 detection using the rapid tests.

\section{ROC analysis (ROC curves)}

The individual rapid tests (DENV1-4, pan-DENV, and ZIKV) were validated using PCR- and/or ELISA-confirmed serum samples containing variable NS1 protein concentrations, resulting in normalized test signal intensities (test signal intensity/background signal intensity) ranging from 0.5 to 5 . On the basis of these data, MATLAB was used to plot and calculate ROC curves, as well as to find the optimal cutoff values for each rapid test type (DENV1-4, pan-DENV, and ZIKV). In practical use, rapid tests with normalized intensity values greater than the cutoff are defined as positive, whereas those with normalized intensity values less than the cutoff are negative. To generate the optimal cutoff values and ROC curves, we used ImageJ to scan each of the test strips, yielding a signal intensity for each test area and also for background in an area of the strip outside of the test and control areas. These normalized intensity values were inputs to a MATLAB script. The script used theoretical cutoffs between 0.5 and 5 and calculated both the sensitivity (number of true positives divided by total confirmed positive values) and specificity (number of true negatives divided by the total confirmed negatives) at each of the theoretical cutoffs. After calculating the sensitivity and specificity at each theoretical cutoff, the optimal cutoff value was defined as the highest sum of sensitivity and specificity. In this way, the optimal cutoff value reflected the optimal sensitivity and specificity performance of the test. To calculate the confidence intervals, we fitted the ImageJ intensity measurements with a generalized linear regression model with binomial distribution and a link logit function using MATLAB's fitglm function. MATLAB's perfcurve function with a bootstrap of 1000 was used to evaluate the linear regression model and calculate the $95 \%$ confidence intervals of the areas under the curve, sensitivity, and specificity. The optimum cutoffs obtained for patient samples were 1.14, 1.18, 1.2, 1.37, 1.19, and 1.08 for lanes $1,2,3,4$, pan-DENV, and ZIKV, respectively.

\section{Statistical analysis}

Origin (OriginLab Corporation) was used to calculate and graph the data. In the box-and-whisker plots, the black $\times$ represents the maximum and minimum measured normalized intensity values, whereas the black box $(\square)$ represents the mean value, and the larger box represents the 25 to $75 \%$ range of the data. Individual colored points represent individual patient samples measured. Unpaired, two-tailed $t$ test or one-way ANOVA was performed to test for statistical significance, as indicated in the figure legends. Individual subject-level data are shown in table S5.

\section{SUPPLEMENTARY MATERIALS}

www.sciencetranslationalmedicine.org/cgi/content/full/9/409/eaan1589/DC1 Materials and Methods

Fig. S1. Stepwise strategy for identifying mAbs that differentiate the closely related NS1 proteins of DENV1-4 and ZIKV.

Fig. S2. Repertoire of antigen-specific antibodies among DENV and ZIKV mAbs. Fig. S3. Limit of detection comparison.

Fig. S4. The Standard Diagnostics DENV NS1 test cross-reacts with ZIKV NS1 protein. Fig. S5. DENV NS1 and ZIKV NS1 detection expressed as days after onset of fever symptoms. Fig. S6. Laboratory-made gold nanoparticles for detecting DENV NS1 in rapid test format. Fig. S7. NS1 detection by rapid tests in secondary infections.

Table S1. Stepwise description of the approaches used to define antibodies that detect and distinguish DENV1-4 NS1 and the ZIKV NS1 proteins.
Table S2. Amino acid homology and identity among DENV NS1 and ZIKV NS1 proteins. Table S3. Matrix of mAb pair trials.

Table S4. List of mAbs used in the rapid tests, relative binding values, and summary of final use in the DENV and ZIKV immunochromatography tests.

Table S5. Individual subject-level data.

References $(47,48)$

\section{REFERENCES AND NOTES}

1. A. Ong, M. Sandar, M. I. Chen, L. Y. Sin, Fatal dengue hemorrhagic fever in adults during a dengue epidemic in Singapore. Int. J. Infect. Dis. 11, 263-267 (2007).

2. D. A. Muller, P. R. Young, The flavivirus NS1 protein: Molecular and structural biology, immunology, role in pathogenesis and application as a diagnostic biomarker. Antiviral Res. 98, 192-208 (2013).

3. A.-C. Andries, V. Duong, S. Ong, S. Ros, A. Sakuntabhai, P. Horwood, P. Dussart, P. Buchy, Evaluation of the performances of six commercial kits designed for dengue NS1 and anti-dengue $\lg M, \lg G$ and $\lg A$ detection in urine and saliva clinical specimens. BMC Infect. Dis. 16, 201 (2016).

4. S. M. Wang, S. D. Sekaran, Early diagnosis of Dengue infection using a commercial Dengue Duo rapid test kit for the detection of NS1, IGM, and IGG. Am. J. Trop. Med. Hyg. 83, 690-695 (2010).

5. J. Lee, H.-Y. Kim, C.-K. Chong, H.-O. Song, Development and clinical evaluation of a highly accurate dengue NS1 rapid test: From the preparation of a soluble NS1 antigen to the construction of an RDT. Diagn. Microbiol. Infect. Dis. 82, 128-134 (2015).

6. V. Tricou, H. T. T. Vu, N. V. N. Quynh, C. V. V. Nguyen, H. T. Tran, J. Farrar, B. Wills, C. P. Simmons, Comparison of two dengue NS1 rapid tests for sensitivity, specificity and relationship to viraemia and antibody responses. BMC Infect. Dis. 10, 142 (2010).

7. V. T. Hang, N. M. Nguyet, D. T. Trung, V. Tricou, S. Yoksan, N. M. Dung, T. Van Ngoc, T. T. Hien, J. Farrar, B. Wills, C. P. Simmons, Diagnostic accuracy of NS1 ELISA and lateral flow rapid tests for dengue sensitivity, specificity and relationship to viraemia and antibody responses. PLOS Negl. Trop. Dis. 3, e360 (2009).

8. S. R. Fry, M. Meyer, M. G. Semple, C. P. Simmons, S. D. Sekaran, J. X. Huang, C. McElnea, C.-Y. Huang, A. Valks, P. R. Young, M. A. Cooper, The diagnostic sensitivity of dengue rapid test assays is significantly enhanced by using a combined antigen and antibody testing approach. PLOS Negl. Trop. Dis. 5, e1199 (2011).

9. L. Osorio, M. Ramirez, A. Bonelo, L. A. Villar, B. Parra, Comparison of the diagnostic accuracy of commercial NS1-based diagnostic tests for early dengue infection. Virol. J. 7, 361 (2010).

10. C. Fernanda Estofolete, A. C. B. Terzian, R. Parreira, A. Esteves, L. Hardman, G. V. Greque, P. Rahal, M. L. Nogueira, Clinical and laboratory profile of Zika virus infection in dengue suspected patients: A case series. J. Clin. Virol. 81, 25-30 (2016).

11. ZIKV Detect IgM Capture ELISA by InBios International Inc: FDA Safety CommunicationWait for Confirmatory Test Results Before Making Patient Management Decisions, in MedWatch: The FDA Safety Information and Adverse Event Reporting Program (2016); www.fda.gov/Safety/MedWatch/SafetyInformation/SafetyAlertsforHumanMedicalProducts/ ucm534538.htm.

12. B. O'Farrell, in The Immunoassay Handbook, D. Wild, R. John, C. Sheehan, S. Binder, J. He, Eds. (Elsevier Ltd., 2013), pp. 89-107.

13. W. C. Brown, D. L. Akey, J. R. Konwerski, J. T. Tarrasch, G. Skiniotis, R. J. Kuhn, J. L. Smith, Extended surface for membrane association in Zika virus NS1 structure. Nat. Struct. Mol. Biol. 23, 865-867 (2016).

14. D. L. Akey, W. C. Brown, S. Dutta, J. Konwerski, J. Jose, T. J. Jurkiw, J. DelProposto, C. M. Ogata, G. Skiniotis, R. J. Kuhn, J. L. Smith, Flavivirus NS1 structures reveal surfaces for associations with membranes and the immune system. Science 343, 881-885 (2014).

15. A. K. I. Falconar, P. R. Young, Production of dimer-specific and dengue virus group crossreactive mouse monoclonal antibodies to the dengue 2 virus non-structural glycoprotein NS1. J. Gen. Virol. 72, 961-965 (1991).

16. J. J. Waggoner, L. Gresh, M. J. Vargas, G. Ballesteros, Y. Tellez, K. J. Soda, M. K. Sahoo, A. Nuñez, A. Balmaseda, E. Harris, B. A. Pinsky, Viremia and clinical presentation in Nicaraguan patients infected with Zika virus, chikungunya virus, and dengue virus. Clin. Infect. Dis. 63, 1584-1590 (2016).

17. E. D'Ortenzio, S. Matheron, X. de Lamballerie, B. Hubert, G. Piorkowski, M. Maquart, D. Descamps, F. Damond, Y. Yazdanpanah, I. Leparc-Goffart, Evidence of sexual transmission of Zika virus. N. Engl. J. Med. 374, 2195-2198 (2016).

18. E. M. Korhonen, E. Huhtamo, A.-M. K. Virtala, A. Kantele, O. Vapalahti, Approach to noninvasive sampling in dengue diagnostics: Exploring virus and NS1 antigen detection in saliva and urine of travelers with dengue. J. Clin. Virol. 61, 353-358 (2014).

19. A. Chuansumrit, W. Chaiyaratana, K. Tangnararatchakit, S. Yoksan, M. Flamand, A. Sakuntabhai, Dengue nonstructural protein 1 antigen in the urine as a rapid and convenient diagnostic test during the febrile stage in patients with dengue infection Diagn. Microbiol. Infect. Dis. 71, 467-469 (2011). 
20. V. Tricou, N. N. Minh, J. Farrar, H. T. Tran, C. P. Simmons, Kinetics of viremia and NS1 antigenemia are shaped by immune status and virus serotype in adults with dengue. PLOS Negl. Trop. Dis5, e1309 (2011).

21. A. J. Hirsch, J. L. Smith, N. N. Haese, R. M. Broeckel, C. J. Parkins, C. Kreklywich, V. R. Defilippis, M. Denton, P. P. Smith, W. B. Messer, L. M. A. Colgin, R. M. Ducore, P. L. Grigsby, J. D. Hennebold, T. Swanson, A. W. Legasse, M. K. Axthelm, R. MacAllister, C. A. Wiley, J. A. Nelson, D. N. Streblow, Zika Virus infection of rhesus macaques leads to viral persistence in multiple tissues. PLOS Pathog.13, e1006219 (2017).

22. S. Bhatt, P. W. Gething, O. J. Brady, J. P. Messina, A. W. Farlow, C. L. Moyes, J. M. Drake, J. S. Brownstein, A. G. Hoen, O. Sankoh, M. F. Myers, D. B. George, T. Jaenisch, G. R. W. Wint, C. P. Simmons, T. W. Scott, J. J. Farrar, S. I. Hay, The global distribution and burden of dengue. Nature 496, 504-507 (2013).

23. H. Kettler, K. White, S. Hawkes, in WHO/TDR (World Health Organization, 2004).

24. R. W. Peeling, D. Mabey, Point-of-care tests for diagnosing infections in the developing world. Clin. Microbiol. Infect.16, 1062-1069 (2010).

25. A. Nisalak, T. P. Endy, S. Nimmannitya, S. Kalayanarooj, U. Thisayakorn, R. M. Scott D. S. Burke, C. H. Hoke, B. L. Innis, D. W. Vaughn, Serotype-specific dengue virus circulation and dengue disease in Bangkok, Thailand from 1973 to 1999.Am. J. Trop. Med Hyg. 68, 191-202 (2003).

26. B. M. Forshey, R. C. Reiner, S. Olkowski, A. C. Morrison, A. Espinoza, K. C. Long S. Vilcarromero, W. Casanova, H. J. Wearing, E. S. Halsey, T. J. Kochel, T. W. Scott, S. T. Stoddard, Incomplete protection against dengue virus type 2 re-infection in Peru. PLOS Negl. Trop. Dis.10, e0004398 (2016).

27. L. Villar, G. H. Dayan, J. L. Arredondo-García, D. M. Rivera, R. Cunha, C. Deseda, H. Reynales, M. S. Costa, J. O. Morales-Ramírez, G. Carrasquilla, L. C. Rey, R. Dietze, K. Luz, E. Rivas, M. C. Miranda Montoya, M. Cortés Supelano, B. Zambrano, E. Langevin M. Boaz, N. Tornieporth, M. Saville, F. Noriega; CYD15 Study Group, Efficacy of a tetravalent dengue vaccine in children in Latin America. N. Engl. J. Med.372, 113-123 (2015).

28. G. Kuno, G.-J. J. Chang, K. R. Tsuchiya, N. Karabatsos, C. B. Cropp, Phylogeny of the genus Flavivirus J. Virol. 72, 73-83 (1998).

29. Y. Saito, M. L. Moi, A. Kotaki, M. Ikeda, S. Tajima, H. Shiba, K. Hosono, M. Saijo, I. Kurane, T. Takasaki, Detecting dengue virus nonstructural protein 1 (NS1) in urine samples using ELISA for the diagnosis of dengue virus infection.Jpn. J. Infect. Dis.68, 455-460 (2015).

30. M. D. Abràmoff, P. J. Magelhães, S. J. Ram, Image processing with ImageJ.Biophotonics Int. 11, 36-42 (2004).

31. C. H. Tyrrell, "A lateral flow smart phone image analysis diagnostic" (2013); https://doi.org/10.15368/theses.2013.165.

32. R. W. Peeling, H. Artsob, J. L. Pelegrino, P. Buchy, M. J. Cardosa, S. Devi, D. A. Enria, J. Farrar, D. J. Gubler, M. G. Guzman, S. B. Halstead, E. Hunsperger, S. Kliks, H. S. Margolis, C. M. Nathanson, V. C. Nguyen, N. Rizzo, S. Vázquez, S. Yoksan, Evaluation of diagnostic tests: Dengue. Nat. Rev. Microbiol.8, S30-S38 (2010).

33. R. K. Miller, N. Zeuch, Machine Vision(Springer Science \& Business Media, 1989).

34. A.-C. Andries, V. Duong, C. Ngan, S. Ong, R. Huy, K. K. Sroin, V. Te, B. Y, P. Lorn Try, P. Buchy, Field evaluation and impact on clinical management of a rapid diagnostic kit that detects dengue NS1, IgM and IgG. PLOS Negl. Trop. Dis6, e1993 (2012).

35. A.-C. Andries, V. Duong, S. Ly, J. Cappelle, K. S. Kim, P. Lorn Try, S. Ros, S. Ong, R. Huy, P. Horwood, M. Flamand, A. Sakuntabhai, A. Tarantola, P. Buchy, Value of routine dengue diagnostic tests in urine and saliva specimens. PLOS Negl. Trop. Dis.9, e0004100 (2015).

36. S. D. Blacksell, R. G. Jarman, M. S. Bailey, A. Tanganuchitcharnchai, K. Jenjaroen, R. V. Gibbons, D. H. Paris, R. Premaratna, H. J. de Silva, D. G. Lalloo, N. P. J. Day, Evaluation of six commercial point-of-care tests for diagnosis of acute dengue infections: The need for combining NS1 antigen and IgM/lgG antibody detection to achieve acceptable levels of accuracy. Clin. Vaccine Immunol.18, 2095-2101 (2011).

37. A. M. Bingham, M. Cone, V. Mock, L. Heberlein-Larson, D. Stanek, C. Blackmore, A. Likos, Comparison of test results for Zika virus RNA in urine, serum, and saliva specimens from persons with travel-associated Zika virus disease-Florida, 2016, MMWR Morb. Mortal. Wkly. Rep.65 (2016); https://espanol.cdc.gov/enes/mmwr/volumes/65/wr/

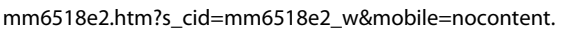

38. D. A. Muller, A. C. I. Depelsenaire, P. R. Young, Clinical and laboratory diagnosis of dengue virus infection. J. Infect. Dis.215, S89-S95 (2017).

39. M. S. Bhamla, B. Benson, C. Chai, G. Katsikis, A. Johri, M. Prakash, Hand-powered ultralowcost paper centrifuge. Nat. Biomed. Eng.1, 0009 (2017).

40. R. Vivek, S. F. Ahamed, S. Kotabagi, A. Chandele, I. Khanna, N. Khanna, K. Nayak, M. Dias, M.-K. Kaja, A. Shet, Evaluation of a pan-serotype point-of-care rapid diagnostic assay for accurate detection of acute dengue infection. Diagn. Microbiol. Infect. Dis87, 229-234 (2017).

41. B. Zhang, B. A. Pinsky, J. S. Ananta, S. Zhao, S. Arulkumar, H. Wan, M. K. Sahoo, J. Abeynayake, J. J. Waggoner, C. Hopes, M. Tang, H. Dai, Diagnosis of Zika virus infection on a nanotechnology platform. Nat. Med. 23, 543-550 (2017)
42. K. Pardee, A. A. Green, M. K. Takahashi, D. Braff, G. Lambert, J. W. Lee, T. Ferrante, D. Ma, N. Donghia, M. Fan, N. M. Daringer, I. Bosch, D. M. Dudley, D. H. OConnor, L. Gehrke, J. J. Collins, Rapid, low-cost detection of Zika virus using programmable biomolecular components. Cell 165, 1255-1266 (2016).

43. R. S. Lanciotti, C. H. Calisher, D. J. Gubler, G.-J. Chang, A. V. Vorndam, Rapid detection and typing of dengue viruses from clinical samples by using reverse transcriptase-polymerase chain reaction. J. Clin. Microbiol.30, 545-551 (1992).

44. A. Becerra, R. V. Warke, K. Xhaja, B. Evans, J. Evans, K. Martin, N. de Bosch, A. L. Rothman, I. Bosch, Increased activity ofi ndoleamine 2,3-dioxygenase in serum from acutely infected dengue patients linked to gamma interferon antiviral function.J. Gen. Virol.90, 810-817 (2009).

45. A. Becerra, R. V. Warke, K. Martin, K. Xhaja, N. de Bosch, A. L. Rothman, I. Bosch, Gene expression profiling of dengue infected human primary cells identifies secreted mediators in vivo. J. Med. Virol.81, 1403-1411 (2009).

46. N. Lukman, G. Salim, H. Kosasih, N. H. Susanto, I. Parwati, S. Fitri, B. Alisjahbana, S. Widjaja, M. Williams, Comparison of the hemagglutination inhibition test and IgG ELISA in categorizing primary and secondary dengue infections based on the plaque reduction neutralization test. Biomed. Res. Int.2016, 5253842 (2016).

47. C.-L. Kao, C.-C. King, D.-Y. Chao, H.-L. Wu, G.-J. J. Chang, Laboratory diagnosis of dengue virus infection: Current and future perspectives in clinical diagnosis and public health J. Microbiol. Immunol. Infect38, 5-16 (2005).

48. S. F. Altschul, T. L. Madden, A. A. Schäffer, J. Zhang, Z. Zhang, W. Miller, D. J. Lipmann, Gapped BLAST and PSI-BLAST: A new generation of protein database search programs. Nucleic Acids Res25, 3389-3402 (1997).

Acknowledgments: We thank B. Baker and P. Regan of the Winchester Engineering and Analytic Center for encouraging the development of this project and for access to equipment and materials, M. Diamond (Washington University, St. Louis) for providing valuable advice as well as mAbs for the initial stages of the work, and J. Munoz (CDC, Puerto Rico) for participating in important discussions and providing access to clinical samples during the early stages of the rapid test development. Peptide arrays were obtained through the NIH Biodefense and Emerging Infections Research Resources Repository, National Institute of Allergy and Infectious Diseases, NIH. We thank M. Zillmann, K. Mann, M. Holstein, M. Higson, C. Gillespie, and P. Kumpey from MilliporeSigma for assistance with the production, purification, and detection antibodies used in this study. We thank A. Versiani for mapping the epitopes for antibody 130 and confirming epitopes for antibody 110 . R. Lodha and S. K. Sabra (Department of Paediatrics, All India Institute of Medical Sciences, New Delhi) were instrumental in obtaining human serum samples. N.d.B. thanks the Banco de Sangre stafffor sample collection and characterization. Funding: F.A.B., M.M.T., A.C.B.T., and P.C.d.-S. received funding from the National Council for Scientific and Technological Development of Brazil and from the Foundation for Research Support of the State of Rio de Janeiro, Brazil (FAPERJ). A.M.B.d.F. was supported by the European Unior's Horizon 2020 program under grant agreement numbers 734857 and 734584 . E.T.A.M. was supported by Programa Pesquisa para o SUS-Fundação de Amparo à Ciência e Tecnologia (PPSUS-FACEPE) project APQ-0302-4.01/13 of the Cura Zika Alliance, University of Pittsburgh. G.R.M. received research support from the Department of Biotechnology, Government of India (sanction no. BT/PR5132/MED/15/84/2012) and Wellcome Trust/DBT India Alliance (IA/S/14/1/501291). L.G. received support from the U.S. Public Health Service (grant AI100190). M.L.N. received support from Fundação de Amparo à Pesquisa do Estado de São Paulo (FAPESP) (grants 13/21719-3 and 16/15021-1). L.V. received support from the Science, Technology and Innovation Fund, ColombiaFondo de Ciencia, Tecnología e Innovación of Sistema General de Regalías (BPIN 2013000100011) and the AEDES Network. H.d.P. received support from a La Caixa Fellowship, a Rafael del Pino Foundation, a Broshy Fellowship, an MIT-Tata Center for Technology and Design fellowship, and an MIF Singapore University of Technology and Design International Design Center grant. M. Benioff and L. Benioffprovided support toward obtaining commercially available Zika samples. Author contributions: Project design and conception: L.G., I.B., J.G.-M., and K.H.-S.; characterization of the mAbs and development of the rapid tests, as well as testing the rapid tests with rNS1 proteins and supernatants from Vero cells infected with DENV or ZIKV and running the rapid tests with patient sera: L.G., I.B., H.d.P., J.G.-M., K.H.-S., M.C.-C., M.H., F.P.-C., D.S., M.O., E.P., A.D., D.F., H.M., C.-W.Y., J.T., and C.C.; collection and characterization of clinical serum samples: F.P.-C., C.F.N., D.M.S., M.G.-R., R.V.W., L.S.R., M.M.T., R.P.A., J.E.M.-M., J.E.L., M.L.N., T.E.C., A.C.B.T., P.T.B., F.A.B., M.R.O.T., T.M.L.S., A.M.B.d.F., P.C.d.S., E.T.A.M., T.M., F.J.D., B.N.R., K.M., S.M., Y.R.V., G.B.-L., A.V., J.C.-N., A.S.C., D.O., M.L., M.S., G.R.M., N.d.B., E.J.A., and L.V.; image analysis and software development: H.d.P. and J.G.-M.; writing the manuscript: L.G., I.B., H.d.P., K.H.-S.; all authors contributed to manuscript editing. Competing interests: R.V.W., HiMedia, has applied to license mAb technology from the MIT. I.B., K.H.-S, J.G.-M., and L.G. are inventors on patent US9488613 B2 filed by MIT that covers devices and methods for multiplexed diagnostics. I.B. K.H.-S., J.G.-M., H.d.P., and L.G. are inventors on MIT case number 18262 (pending), which covers anti-DENV NS1 protein mAbs. I.B., K.H.-S., J.G.-M., H.d.P., and L.G. are inventors on MIT case number 18671 (pending), which covers anti-ZIKV NS1 protein mAbs and pairwise detection of ZIKV NS1 protein. Data and materials availability: mAbs are available in limited 
quantities from the Gehrke Laboratory at MIT under a material transfer agreement with the university. Inquiries about the availability of antibodies should be directed to L.G. (lgehrke@mit.edu).

Submitted 14 March 2017

Resubmitted 2 June 2017

Accepted 8 September 2017

Published 27 September 2017

10.1126/scitranslmed.aan 1589
Citation: I. Bosch, H. de Puig, M. Hiley, M. Carré-Camps, F. Perdomo-Celis, C. F. Narváez, D. M. Salgado, D. Senthoor, M. O'Grady, E. Phillips, A. Durbin, D. Fandos, H. Miyazaki, C.- W. Yen, M. Gélvez-Ramírez, R. V. Warke, L. S. Ribeiro, M. M. Teixeira, R. P. Almeida, J. E. Muñóz-Medina, J. E. Ludert, M. L. Nogueira, T. E. Colombo, A. C. B. Terzian, P. T. Bozza, A. S. Calheiros, Y. R. Vieira, G. Barbosa-Lima, A. Vizzoni, J. Cerbino-Neto, F. A. Bozza, T. M. L. Souza, M. R. O. Trugilho, A. M. B. de Filippis, P. C. de Sequeira, E. T. A. Marques, T. Magalhaes, F. J. Díaz, B. N. Restrepo, K. Marín, S. Mattar, D. Olson, E. J. Asturias, M. Lucera, M. Singla, G. R. Medigeshi, N. de Bosch, J. Tam, J. Gómez-Márquez, C. Clavet, L. Villar, K. Hamad-Schifferli, L. Gehrke, Rapid antigen tests for dengue virus serotypes and Zika virus in patient serum. Sci. Transl. Med. 9, eaan1589 (2017). 


\title{
Science Translational Medicine
}

\section{Rapid antigen tests for dengue virus serotypes and Zika virus in patient serum}

Irene Bosch, Helena de Puig, Megan Hiley, Marc Carré-Camps, Federico Perdomo-Celis, Carlos F. Narváez, Doris M. Salgado, Dewahar Senthoor, Madeline O'Grady, Elizabeth Phillips, Ann Durbin, Diana Fandos, Hikaru Miyazaki, Chun-Wan Yen, Margarita Gélvez-Ramírez, Rajas V. Warke, Lucas S. Ribeiro, Mauro M. Teixeira, Roque P. Almeida, José E. Muñóz-Medina, Juan E. Ludert, Mauricio L. Nogueira, Tatiana E. Colombo, Ana C. B. Terzian, Patricia T. Bozza, Andrea S. Calheiros, Yasmine R. Vieira, Giselle Barbosa-Lima, Alexandre Vizzoni, José Cerbino-Neto, Fernando A. Bozza, Thiago M. L. Souza, Monique R. O. Trugilho, Ana M. B. de Filippis, Patricia C. de Sequeira, Ernesto T. A. Marques, Tereza Magalhaes, Francisco J. Díaz, Berta N. Restrepo, Katerine Marín, Salim Mattar, Daniel Olson, Edwin J. Asturias, Mark Lucera, Mohit Singla, Guruprasad R. Medigeshi, Norma de Bosch, Justina Tam, Jose Gómez-Márquez, Charles Clavet, Luis Villar, Kimberly Hamad-Schifferli and Lee Gehrke

Sci Transl Med 9, eaan1589.

DOI: 10.1126/scitranslmed.aan1589

\begin{abstract}
Distinguishing dengue from Zika
More than mere summer pests, mosquitoes can transmit viruses, such as dengue and Zika. Diagnosing infections of these related flaviviruses can be difficult because of cross-reactivity in diagnostic tests. Bosch et al. developed monoclonal antibodies to detect viral nonstructural 1 (NS1) protein antigens specific to dengue and Zika. Incorporating the antibodies into an immunochromatography format yielded a rapid diagnostic assay that produces a visual readout in the presence of NS1. The assay identified the four dengue serotypes and Zika viral infections without cross-reaction when testing human serum samples from endemic areas in Central and South
\end{abstract} America and India. This approach could be useful for developing rapid diagnostics for other emerging pathogens.

ARTICLE TOOLS

SUPPLEMENTARY MATERIALS

RELATED CONTENT

REFERENCES

PERMISSIONS http://stm.sciencemag.org/content/9/409/eaan1589

http://stm.sciencemag.org/content/suppl/2017/09/25/9.409.eaan1589.DC1

\begin{abstract}
http://stm.sciencemag.org/content/scitransmed/7/278/278ra35.full http://stm.sciencemag.org/content/scitransmed/7/304/304ra141.full http://stm.sciencemag.org/content/scitransmed/7/304/304fs37.full http://stm.sciencemag.org/content/scitransmed/9/388/eaag0538.full http://stm.sciencemag.org/content/scitransmed/8/369/369ra179.full http://stm.sciencemag.org/content/scitransmed/3/114/114ra128.full http://stm.sciencemag.org/content/scitransmed/8/330/330ed2.full http://stm.sciencemag.org/content/scitransmed/8/330/330ra36.full http://stm.sciencemag.org/content/scitransmed/9/410/eaan8184.full
\end{abstract}

This article cites 42 articles, 6 of which you can access for free http://stm.sciencemag.org/content/9/409/eaan1589\#BIBL

http://www.sciencemag.org/help/reprints-and-permissions

Use of this article is subject to the Terms of Service

Science Translational Medicine (ISSN 1946-6242) is published by the American Association for the Advancement of Science, 1200 New York Avenue NW, Washington, DC 20005. 2017 (C The Authors, some rights reserved; exclusive licensee American Association for the Advancement of Science. No claim to original U.S. Government Works. The title Science Translational Medicine is a registered trademark of AAAS. 